\title{
RESEARCH
}

Open Access

\section{Dental caries in primary and permanent teeth in children's worldwide, 1995 to 2019: a systematic review and meta- analysis}

Mohsen Kazeminia ${ }^{1}$, Alireza Abdi ${ }^{1}$, Shamarina Shohaimi ${ }^{2}$, Rostam Jalali ${ }^{1}$, Aliakbar Vaisi-Raygani ${ }^{1}$, Nader Salari ${ }^{3{ }^{*}}$ and Masoud Mohammadi ${ }^{1 *}$ (D)

\begin{abstract}
Background: Early childhood caries (ECC) is a type of dental caries in the teeth of infants and children that is represented as one of the most prevalent dental problems in this period. Various studies have reported different types of prevalence of dental caries in primary and permanent teeth in children worldwide. However, there has been no comprehensive study to summarize the results of these studies in general, so this study aimed to determine the prevalence of dental caries in primary and permanent teeth in children in different continents of the world during a systematic review and meta-analysis.

Methods: In this review study, articles were extracted by searching in the national and international databases of SID, Maglran, IranMedex, IranDoc, Cochrane, Embase, ScienceDirect, Scopus, PubMed, and Web of Science (ISI) between 1995 and December 2019. Random effects model was used for analysis and heterogeneity of studies was evaluated by using the $I^{2}$ index. Data were analyzed by using the Comprehensive Meta-Analysis (Version 2) software.

Findings: In this study, a total of 164 articles (81 articles on the prevalence of dental caries in primary teeth and 83 articles on the prevalence of dental caries in permanent teeth) were entered the meta-analysis. The prevalence of dental caries in primary teeth in children in the world with a sample size of 80,405 was 46.2\% (95\% Cl: 41.6-50.8\%), and the prevalence of dental caries in permanent teeth in children in the world with a sample size of 1,454,871 was 53.8\% (95\% Cl: 50-57.5\%). Regarding the heterogeneity on the basis of meta-regression analysis, there was a significant difference in the prevalence of dental caries in primary and permanent teeth in children in different continents of the world. With increasing the sample size and the year of study, dental caries in primary teeth increased and in permanent teeth decreased.

(Continued on next page)
\end{abstract}

\footnotetext{
* Correspondence: n_s_514@yahoo.com;

Masoud.mohammadi1989@yahoo.com

${ }^{3}$ Department of Biostatistics, School of Health, Kermanshah University of Medical Sciences, Kermanshah, Iran

'Department of Nursing, School of Nursing and Midwifery, Kermanshah University of Medical Sciences, Kermanshah, Iran

Full list of author information is available at the end of the article
}

(C) The Author(s). 2020 Open Access This article is licensed under a Creative Commons Attribution 4.0 International License, which permits use, sharing, adaptation, distribution and reproduction in any medium or format, as long as you give appropriate credit to the original author(s) and the source, provide a link to the Creative Commons licence, and indicate if changes were made. The images or other third party material in this article are included in the article's Creative Commons licence, unless indicated otherwise in a credit line to the material. If material is not included in the article's Creative Commons licence and your intended use is not permitted by statutory regulation or exceeds the permitted use, you will need to obtain permission directly from the copyright holder. To view a copy of this licence, visit http://creativecommons.org/licenses/by/4.0/ The Creative Commons Public Domain Dedication waiver (http://creativecommons.org/publicdomain/zero/1.0/) applies to the data made available in this article, unless otherwise stated in a credit line to the data. 
(Continued from previous page)

Conclusion: The results of this study showed that the prevalence of primary and permanent dental caries in children in the world was found to be high. Therefore, appropriate strategies should be implemented to improve the aforementioned situation and to troubleshoot and monitor at all levels by providing feedback to hospitals.

Keywords: Caries, Tooth, Primary and permanent, Prevalence, Meta-analysis

\section{Background}

Early childhood caries (ECC) is a type of dental caries in the teeth of infants and children that is represented as one of the most prevalent dental problems in this period [1] which can lead to pain, infection, interference with eating, increased risk of new dental caries in primary and permanent teeth, and, ultimately, worse effects on the eruption of permanent teeth [2]. These manifestations can range from demineralization to loss of tooth structure or complete destruction of the crown, a process of dynamic and active decay characterized by various periods of destruction and repair [3].

According to the American Academy of Dentistry, early childhood caries (ECC) is defined as "the presence of 1 or more decayed (non-cavitated or cavitated lesions), missing (due to caries), or filled tooth surfaces in any primary tooth" in children [1]. Overall, $50 \%$ of children have one or more decayed primary teeth by the end of toddler age, but the importance of these teeth should not be overlooked, because, as has been said, healthy teeth in childhood have an important role in the eruption of healthy permanent teeth, healthy nutrition, and one's aesthetic appearance [2, 3]. Factors such as malnutrition, genetic predisposition, poor health performance, specific eating habits, the presence of organisms affecting tooth decay such as streptococci, and fluoride and vitamin D deficiency, excessive sugar consumption and prolonged bottle-feeding, and other factors such as age, gender and place of residence of children are effective in causing tooth decay [4].

The World Health Organization (WHO) has represented the early childhood caries as a worldwide problem with a prevalence of between 60 and 90\% [5]. According to the statistics provided by the European countries, $61 \%$ of children aged 6 to 12 years have at least one decayed tooth, and due to widespread dental caries in all social classes, this disease can impose a great financial burden on the society [6]. In Iran the mean decay-missing-filled (DMF) index of primary teeth in children aged 3 to 6 years was 1.7 and DMF index of permanent teeth was reported to be 0.2 in 6- to 9-yearold children, 0.9 to 1.5 in 12-year-old children, and 3.3 to 4.8 in 9-year-old children [7]. The decay-missingfilled (DMF) index is used as an appropriate measure for the detection of dental caries in the society in which 12year-old children are considered as a target group [8].

Primary teeth begin to erupt in infants' mouths at about 6 months of age, and are completed at age 3 to 5 , including 10 teeth in the maxilla and 10 in the mandible to meet nutritional needs in infancy [6]. Since primary teeth are the basis of permanent teeth, on the one hand, and they have a high susceptibility to caries, on the other hand, these teeth are very important and maintaining their health is considered a serious health concern for children [3,9].

Due to the influence of different factors on the prevalence of primary and permanent dental caries in children and lack of general statistics about this issue worldwide, we decided to review the studies in this area and to statistically analyze the results of these studies to compile a general statistics on the prevalence of dental caries in primary and permanent teeth in children in different continents of the world to open a window to more precise planning to reduce the complications of primary and permanent dental caries in children.

\section{Methods}

In this systematic review and meta-analysis, the prevalence of dental caries in primary and permanent teeth in children was evaluated based on studies conducted between 1995 and December 2019. To this end, articles published in the national databases of SID, MagIran, IranMedex, and IranDoc, and in the international databases of Google scholar, Cochrane, Embase, ScienceDirect, Scopus, PubMed, and Web of Science (ISI) were searched by using Persian and English keywords such as Prevalence, Caries, Rampant caries, Milky tooth, Permanent tooth and Children.

\section{Selection of studies}

Initially, all articles referring to the prevalence of dental caries in primary and permanent teeth in children in the world were collected by the researchers and accepted based on the inclusion and exclusion criteria. The inclusion criteria were observational (non-interventional) studies and their full text availability. For more information, the sources of the articles reviewed were also reviewed for access to other articles. 
Exclusion criteria included irrelevant cases, case reports, interventional studies and other review, casecontrol, cohort, duplication of studies, unclear methodology, and full text unavailability. In order to reduce bias, the articles were searched independently by two researchers, and if they disagreed on a study, the article was reviewed by the group supervisor (blinded about the decision by the first two independent researchers' decision). A total of 180 studies entered the third stage, quality assessment.

Duplicate publication and multiple publications from the same population will be removed using citation management, software EndNote (version X7, for Windows, Thomson Reuters).

\section{Quality assessment of studies}

The quality of the articles was first evaluated on the basis of selected and related items of the 22- item STROBE checklist that could be evaluated in this study (study design, background and literature review, place and time of study, outcome, inclusion criteria, sample size, and statistical analysis) and also mentioned in the previous studies. Articles referring to 6 to 7 criteria were considered as high quality articles, articles that did not mention 2 items and more than 2 items from the seven items were considered as medium and low methodological quality articles, respectively [10]. In the present study, 164 articles with high quality and medium quality were entered the systematic review and meta-analysis, and 16 articles were of poor quality and were excluded.

\section{Data extraction}

All final articles entered into the meta-analysis process were prepared to be extracted by a pre-prepared checklist. The checklist included article title, first author's name, year of publication, place of study, sample size, mean age of sample, prevalence of dental caries in primary and permanent teeth.

\section{Statistical analysis}

Since prevalence has binomial distribution, prevalence variance was calculated using the binomial distribution variance formula, and weighted mean was used to combine the prevalence rate of different studies. In order to evaluate the heterogeneity of the selected studies, $\mathrm{I}^{2}$ test was used (heterogeneity was divided into three classes of less than 25\% (low heterogeneity), 25-75\% (moderate heterogeneity) and more than $75 \%$ (high heterogeneity). Meta-regression analysis was used to investigate the relationship between the prevalence of dental caries with the sample size and the year of study. In order to evaluate the publication error with respect to the large sample size of studies entered the review, the Begg and Mazumdar test at the significant level of 0.1 and its corresponding Funnel plot were used. Sensitivity analysis was used to evaluate the effect of individual studies on the final result. Data were analyzed by using the Comprehensive Meta-Analysis (Version 2) software.

\section{Results}

In this study, all studies conducted on the prevalence of primary and permanent dental caries in children in the world were systematically investigated without time limitation based on the PRISMA guidelines. In the initial search, 2870 articles were identified, form which 164 studies published between 1995 and December 2019 were eventually entered the final analysis (Fig. 1).

The total sample size for the prevalence of primary dental caries was 80,405 and for the prevalence of permanent dental caries was $1,454,871$, given with the mean age of the subjects in each study. The specifications of the selected articles are presented in Tables 1 and 2 .

\section{Heterogeneity and publication bias}

Based on the results of the heterogeneity evaluation test $\left(\mathrm{I}^{2}\right)$, the prevalence of dental caries in primary and permanent teeth was reported to be $\mathrm{I}^{2}: 99.2$ and I2: 99.8, respectively. Due to the heterogeneity of the selected studies, a random effects model was used to combine the studies and jointly estimate the prevalence of dental caries in primary and permanent teeth. The probability of publication bias was evaluated by the Funnel plot and the Begg and Mazumdar tests at a significant level of 0.1 (Figs. 2 and 3), indicating that the publication bias was not statistically significant in the investigation of the prevalence of primary dental caries $(P=0.590)$ and permanent dental caries $(P=0.145)$.

\section{Meta-analysis (primary dental caries)}

According to the results of the study in forest plot, the overall prevalence of dental caries in primary teeth in children in the world was $46.2 \%$ (95\% CI: 41.6-50.8\%) (Fig. 4). The middle point of each line shows the prevalence of primary dental caries in the world for each study, and the rhombic figure shows the prevalence of primary dental caries in the world for all studies.

\section{Meta-analysis (permanent dental caries)}

According to the results of the study in forest plot, the overall prevalence of dental caries in permanent teeth in children in the world was $53.8 \%$ (95\% CI: $50-57.5 \%$ ) (Fig. 5). The middle point of each line 


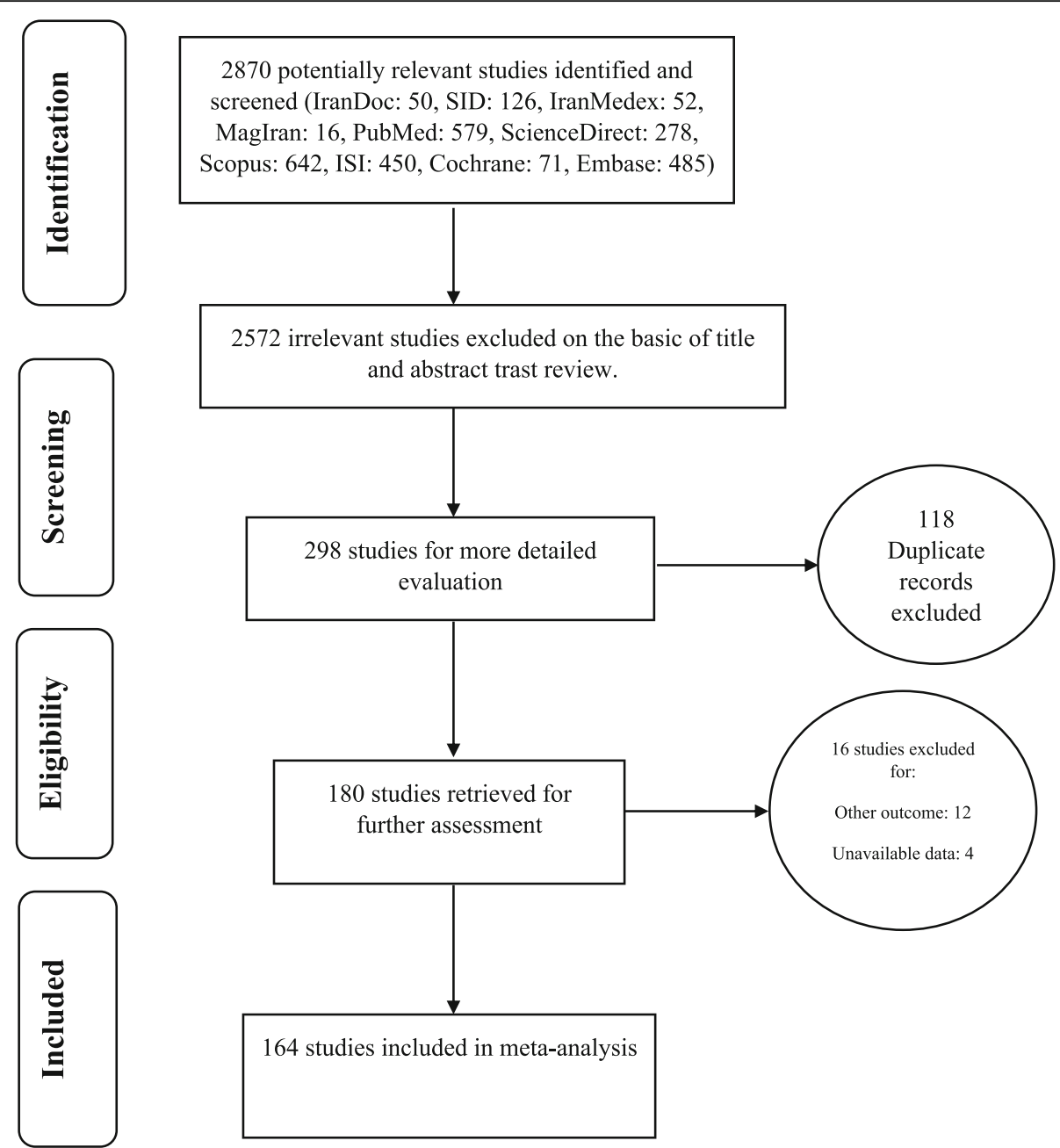

Fig. 1 Flow diagram of study selection

shows the prevalence of permanent dental caries in the world for each study, and the rhombic figure shows the prevalence of permanent dental caries in the world for all studies.

\section{Meta-regression}

The prevalence of primary dental caries was evaluated by meta-regression analysis based on the year of study and the sample size, which reported that with increasing the year of study and the sample size, the prevalence of dental caries in primary teeth increased in both cases and the difference was statistically significant $(\mathrm{P}<0.01)$ (Figs. 6 and 7).

The prevalence of permanent dental caries was evaluated based on the year of study and the sample size, which reported that with increasing the year of study and the sample size, the prevalence of dental caries in permanent teeth decreased in both cases and the difference was statistically significant $(\mathrm{P}<0.01)$ (Figs. 8 and 9 ).

\section{Sub-group analysis}

Also, Table 3 and Fig. 10 report the results of the prevalence of dental caries in primary and permanent teeth in children in different continents. These changes were reported in the continents of Asia, Europe, Africa, USA, and Australia, according to which 100 Asian studies (50 studies on the prevalence of dental caries in primary teeth and 50 studies on the prevalence of dental caries in permanent teeth), 32 European studies (10 studies on the prevalence of dental caries in primary teeth and 22 studies on the prevalence of dental caries in permanent teeth), 21 American studies (14 studies on the prevalence of dental caries in primary teeth and 7 studies on the prevalence of dental caries in permanent teeth), 10 African studies (5 studies on the prevalence of dental 
Table 1 Characteristic of included studies prevalence of tooth decay

\begin{tabular}{|c|c|c|c|c|c|}
\hline Author, year, Reference & Age (years) & Country & Sample size & Prevalence \% & Quality \\
\hline Kalantari, 2014, [11] & $6-7$ & Iran & 400 & 63.5 & High \\
\hline Abedini, 2013, [12] & $2-6$ & Iran & 310 & 48.7 & High \\
\hline Hematyar, 2009, [13] & $3-7$ & Iran & 200 & 63.5 & High \\
\hline Nabipour, 2013, [14] & $3-6$ & Iran & 838 & 71.8 & High \\
\hline Pahlavani, 2008, [15] & $2-6$ & Iran & 414 & 61.6 & High \\
\hline Amiri, 2017, [16] & $4-6$ & Iran & 359 & 87.7 & High \\
\hline Ajami, 2005, [17] & $6-7$ & Iran & 1938 & 76.5 & High \\
\hline Javadinezhad, 2008, [18] & 40.5 Month & Iran & 100 & 77.0 & High \\
\hline Karimi, 2012, [19] & $2-5$ & Iran & 211 & 82.9 & High \\
\hline Amanlou, 2011, [20] & $3-6$ & Iran & 205 & 49.3 & High \\
\hline Toutouni, 2015, [21] & $2-3$ & Iran & 239 & 61.1 & High \\
\hline Bagherian, 2013, [22] & $2-5$ & Iran & 400 & 51.3 & Medium \\
\hline Mohebbi, 2006, [23] & $1-3$ & Iran & 504 & 32.9 & High \\
\hline Ramos-Gomez, 1995, [24] & $<6$ & USA & 220 & 30.0 & High \\
\hline Rosenblatt, 2002, [25] & $1-3$ & USA & 468 & 28.4 & High \\
\hline Rajab, 2001, [26] & $1-5$ & Jordan & 384 & 47.9 & High \\
\hline Douglass, 1999, [27] & $3-4$ & USA & 517 & 37.9 & High \\
\hline Hallett, 1998, [28] & $4-6$ & Australia & 3375 & 37.6 & High \\
\hline Sayegh, 2002, [29] & $4-5$ & Jordan & 1140 & 67.0 & Medium \\
\hline Hallett, 2003, [30] & $4-5$ & Australia & 2474 & 37.6 & High \\
\hline Peressini, 2000, [31] & $3-5$ & Canada & 87 & 19.5 & High \\
\hline Chadwick, 2005, [32] & $2-5$ & UK & 449 & 24.1 & High \\
\hline Schroth, 2015, [33] & $<6$ & Canada & 408 & 53.7 & High \\
\hline Tsai, 1997, [34] & $<6$ & Taiwan & 951 & 55.9 & Medium \\
\hline Mahejabeen, 2006, [35] & $3-5$ & India & 1500 & 54.1 & Medium \\
\hline Du, 2002, [36] & $3-5$ & China & 2014 & 55.3 & High \\
\hline Ferro, 2005, [37] & $3-5$ & Italy & 4198 & 25.0 & High \\
\hline Schroth, 2004, [38] & $1-5$ & USA & 834 & 71.0 & Medium \\
\hline Wyne, 2008, [39] & $3-5$ & Saludi Arabia & 789 & 74.8 & High \\
\hline Lawrence, 2004, [40] & $1-5$ & Canada & 1275 & 72.7 & High \\
\hline Vazquez-Nava, 2005, [41] & $4-5$ & Mexico & 1160 & 17.9 & High \\
\hline Jigjid, 2005, [42] & $1-5$ & Japan & 670 & 71.9 & High \\
\hline Senesombath, 2010, [43] & 36-47 Month & Thailand & 400 & 82.0 & Medium \\
\hline Slabsinskiene, 2003, [44] & 3 & Lithuania & 950 & 50.6 & High \\
\hline Zhou, 2010, [45] & 32 Month & China & 155 & 28.4 & High \\
\hline Rajshekar, 2005, [46] & $1-6$ & India & 500 & 43.4 & Medium \\
\hline Ozer, 2011, [47] & $3-6$ & Turkey & 226 & 49.6 & High \\
\hline Li, 2011, [48] & 36-70 Month & China & 1523 & 56.8 & High \\
\hline Kumarihamy, 2011, [49] & $1-2$ & Sri Lanka & 410 & 32.2 & High \\
\hline Prakash, 2012, [50] & 8-48 Month & India & 1500 & 27.5 & Medium \\
\hline Singh, 2012, [51] & 36-60 Month & India & 717 & 40.0 & High \\
\hline Perera, 2010, [52] & 24-71 Month & Sri Lanka & 410 & 32.2 & Medium \\
\hline Phipps, 2011, [53] & 12-71 Month & India & 8461 & 62.3 & High \\
\hline Parisotto, 2012, [54] & 36-59 Month & Brazil & 351 & 39.9 & High \\
\hline
\end{tabular}


Table 1 Characteristic of included studies prevalence of tooth decay (Continued)

\begin{tabular}{|c|c|c|c|c|c|}
\hline Author, year, Reference & Age (years) & Country & Sample size & Prevalence \% & Quality \\
\hline Zhang, 2012, [55] & 5 & China & 723 & 84.9 & High \\
\hline Tanaka, 2007, [56] & 3 & Japan & 2055 & 20.7 & Medium \\
\hline Colombo, 2019, [57] & 48-71 Month & Italy & 2522 & 38.0 & Medium \\
\hline Agouropoulos-1, 2019, [58] & $<7$ & USA & 175 & 92.6 & High \\
\hline Agouropoulos-2, 2019, [58] & $<7$ & USA & 175 & 53.7 & High \\
\hline Agouropoulos-3, 2019, [58] & $<7$ & USA & 175 & 36.0 & High \\
\hline Musinguzi, 2019, [59] & $3-5$ & Kenya & 432 & 48.1 & High \\
\hline Montes, 2019, [60] & $5-7$ & Brazil & 415 & 42.9 & Medium \\
\hline Boustedt, 2019, [61] & 5 & Sweden & 336 & 13.1 & High \\
\hline Tonpe-1, 2019, [62] & $3-5$ & India & 358 & 2.8 & High \\
\hline Tonpe-2, 2019, [62] & $3-5$ & India & 358 & 4.2 & High \\
\hline Wang, 2019, [63] & 6 & China & 4936 & 87.7 & High \\
\hline Nomura, 2019, [64] & $5-6$ & Myanmar & 187 & 81.3 & Medium \\
\hline Wu, 2019, [65] & $5-6$ & China & 1350 & 51.4 & High \\
\hline Abbass, 2019, [66] & $5-6$ & Egypt & 369 & 4.3 & Medium \\
\hline Goenka, 2018, [67] & $5-7$ & India & 312 & 65.1 & High \\
\hline Chugh, 2018, [68] & 24-61 Month & India & 425 & 47.3 & High \\
\hline Vandana, 2018, [69] & $2-6$ & India & 550 & 38.2 & High \\
\hline Igic, 2018, [70] & $3-6$ & Serbia & 250 & 38.4 & High \\
\hline Kato, 2017, [71] & 3 & Japan & 6315 & 36.0 & High \\
\hline Li, 2017, [72] & $3-5$ & China & 1727 & 78.2 & High \\
\hline Mangla, 2017, [73] & $1-3$ & India & 510 & 21.0 & High \\
\hline Owen, 2017, [74] & $3-5$ & Australia & 623 & 14.1 & High \\
\hline Pal, 2017, [75] & $5-6$ & India & 408 & 46.6 & High \\
\hline Wagne, 2017, [76] & 6.7 & Germany & 512 & 1.8 & High \\
\hline Shah, 2017, [77] & $5-7$ & India & 829 & 33.2 & Medium \\
\hline Yuan, 2017, [78] & 3 & China & 959 & 28.1 & High \\
\hline Jiang, 2017, [79] & $2-5$ & China & 1509 & 71.4 & High \\
\hline Massignan, 2016, [80] & 3.7 & Brazil & 565 & 39.1 & High \\
\hline Koya, 2016, [81] & 24-71 Month & India & 1897 & 42.0 & High \\
\hline Mothupi, 2016, [82] & 4.8 & Africa & 495 & 48.9 & High \\
\hline Alkhtib, 2016, [83] & $4-5$ & Qatari & 250 & 89.2 & High \\
\hline Henry, 2016, [84] & $<3$ & India & 1486 & 40.6 & High \\
\hline Šačić, 2016, [85] & $3-5$ & Bosnia and Herzegovina & 165 & 17.0 & Medium \\
\hline Al-Meedani, 2016, [86] & $3-5$ & Saludi Arabia & 388 & 69.1 & High \\
\hline Elidrissi, 2016, [87] & $3-5$ & Sudan & 553 & 52.4 & Medium \\
\hline Gopal, 2016, [88] & $3-6$ & India & 477 & 27.3 & High \\
\hline
\end{tabular}

caries in primary teeth and 5 studies on the prevalence of dental caries in permanent teeth), and 4 Australian studies (3 studies on the prevalence of dental caries in primary teeth and 1 studies on the prevalence of dental caries in permanent teeth) were included in the metaanalysis. There was a significant difference in the prevalence of dental caries in primary and permanent teeth in different continents (Table 3 and Fig. 10).

\section{Cumulative meta-analysis}

A cumulative meta-analysis of the included studies was performed based primary and permanent dental caries. 
Table 2 Characteristic of included studies Prevalence of permanent dental caries

\begin{tabular}{|c|c|c|c|c|c|}
\hline Author, year, Reference & Age (years) & Country & Sample size & Prevalence \% & Quality \\
\hline Aghighi, 2010, [89] & $6-15$ & Iran & 4666 & 66.3 & High \\
\hline Mortazavi, 1997, [90] & $6-9$ & Iran & 220 & 65.5 & High \\
\hline Asdagh, 2015, [91] & $6-12$ & Iran & 847 & 79.7 & Medium \\
\hline Memar, 1999, [92] & 12 & Iran & 439 & 84.3 & High \\
\hline Javadi nejad, 2006, [93] & 12 & Iran & 340 & 82.1 & High \\
\hline Sadeghi, 2007, [94] & 12 & Iran & 563 & 68.6 & High \\
\hline Yousofi, 2015, [95] & $7-12$ & Iran & 460 & 89.8 & High \\
\hline Eskandarizadeh, 2015, [96] & $6-12$ & Iran & 15,369 & 79.5 & High \\
\hline Mossaheb, 2011, [97] & $6-11$ & Iran & 203 & 82.3 & High \\
\hline Qin, 2019, [98] & $10-12$ & China & 5057 & 39.2 & High \\
\hline Alshehhi, 2019, [99] & 8.1 & United Arab Emirates & 62 & 58.1 & High \\
\hline Cheng, 2019, [100] & 10.3 & China & $1,196,004$ & 41.1 & High \\
\hline Villanueva-Gutiérrez, 2019, [101] & 9 & Spain & 686 & 35.4 & High \\
\hline Lešić, 2019, [102] & $6-15$ & Croatia & 1589 & 50.0 & High \\
\hline Mohd Nor, 2019, [103] & 12 & Malaysia & 595 & 74.3 & High \\
\hline Vanvitelli, 2019, [104] & $8-10$ & Italy & 530 & 29.1 & Medium \\
\hline Obregón-Rodríguez-1, 2019, [105] & 12 & Spain & 1045 & 25.5 & High \\
\hline Obregón-Rodríguez-2, 2019, [105] & 15 & Spain & 783 & 26.2 & High \\
\hline Mimoza, 2019, [106] & $7-10$ & Italy & 398 & 28.4 & Medium \\
\hline Abbass, 2019, [66] & $6-12$ & Egypt & 369 & 27.9 & High \\
\hline Aldossary, 2018, [107] & $6-9$ & Saudi Arabia & 1844 & 95.0 & High \\
\hline Goenka, 2018, [67] & $8-10$ & India & 353 & 56.7 & High \\
\hline Ballouk, 2019, [108] & $8-12$ & Syria & 1500 & 79.1 & High \\
\hline Alhabdan, 2018, [109] & $6-8$ & Saudi Arabia & 578 & 82.9 & High \\
\hline Konde, 2018, [110] & 12 & India & 1000 & 13.6 & High \\
\hline Solis-Riggioni, 2018, [111] & $8-15$ & Costa Rica & 201 & 35.8 & High \\
\hline Musa, 2018, [112] & $7-11$ & China & 24,521 & 32.4 & High \\
\hline Dutra, 2018, [113] & $8-12$ & Brazil & 1211 & 32.4 & High \\
\hline Al-Akwa, 2018, [114] & $6-12$ & Yemen & 17,599 & 67.6 & Medium \\
\hline Cruz, 2018, [115] & $11-12$ & Brazil & 184 & 34.2 & High \\
\hline Andegiorgish, 2017, [116] & 12 & Eritrea & 225 & 77.8 & High \\
\hline Alwayli, 2017, [117] & $6-9$ & Saudi Arabia & 17,891 & 64.6 & High \\
\hline Dobbiani-1, 2012, [118] & 10 & Italy & 400 & 44.0 & Medium \\
\hline Dobbiani-2, 2013, [118] & 10 & Italy & 400 & 18.5 & Medium \\
\hline Maran, 2017, [119] & $6-12$ & India & 1204 & 73.2 & High \\
\hline Shah, 2017, [77] & $12-15$ & India & 829 & 31.4 & High \\
\hline Kim, 2017, [120] & $6-11$ & Korea & 514 & 49.4 & High \\
\hline Sköld, 2016, [121] & 13 & Sweden & 758 & 2.6 & High \\
\hline Plaka, 2017, [122] & $12-15$ & India & 193 & 36.3 & High \\
\hline Hiremath, 2016, [123] & $6-11$ & India & 13,200 & 78.9 & High \\
\hline Kottayi, 2016, [124] & $12-15$ & India & 2000 & 3.9 & Medium \\
\hline Ponnudurai, 2016, [125] & $6-14$ & India & 2796 & 68.8 & High \\
\hline Djossou, 2013, [126] & $6-15$ & Benin & 497 & 49.7 & High \\
\hline Weusmann, 2015, [127] & 8 & Germany & 25,020 & 60.9 & High \\
\hline
\end{tabular}


Table 2 Characteristic of included studies Prevalence of permanent dental caries (Continued)

\begin{tabular}{|c|c|c|c|c|c|}
\hline Author, year, Reference & Age (years) & Country & Sample size & Prevalence \% & Quality \\
\hline Goel-1, 2015, [128] & 12 & India & 992 & 34.3 & Medium \\
\hline Goel-2, 2015, [128] & 15 & India & 992 & 46.5 & High \\
\hline Farooqi, 2014, [129] & $6-9$ & Saudi Arabia & 711 & 73.0 & High \\
\hline Arora, 2014, [130] & 12 & India & 100 & 57.0 & High \\
\hline Sukhabogi-1, 2014, [131] & 12 & India & 924 & 39.9 & Medium \\
\hline Sukhabogi-2, 2014, [131] & $15-$ & India & 951 & 46.7 & Medium \\
\hline Al-Darwish, 2014, [132] & $12-14$ & Qatar & 2113 & 85.0 & High \\
\hline Aidara, 2014, [133] & $12-15$ & Senegal & 677 & 96.0 & High \\
\hline Ingle, 2014, [134] & $12-15$ & India & 1400 & 53.0 & Medium \\
\hline Sofola, 2000, [135] & $6-12$ & Nigeria & 513 & 16.6 & High \\
\hline Das, 2013, [136] & $6-14$ & West Bengal & 1764 & 28.1 & High \\
\hline Mahfouz-1, 2013, [137] & 12 & Palestine & 677 & 40.6 & High \\
\hline Mahfouz-2, 2013, [137] & 13 & Palestine & 677 & 41.8 & High \\
\hline Mahfouz-3, 2013, [137] & 14 & Palestine & 677 & 60.4 & High \\
\hline Riziwaguli, 2013, [138] & $7-9$ & China & 1600 & 26.5 & Medium \\
\hline Joshi, 2013, [139] & $6-12$ & India & 1600 & 69.1 & Medium \\
\hline Pieper, 2009, [140] & 12 & Germany & 30,943 & 72.7 & High \\
\hline Yengopal, 2012, [141] & 10.5 & Africa & 882 & 27.6 & High \\
\hline Murthy, 2014, [142] & $12-15$ & India & 1452 & 57.9 & High \\
\hline Koposova, 2013, [143] & 12 & Russia & 590 & 68.0 & High \\
\hline Dixit, 2013, [144] & $12-13$ & Nepal & 361 & 41.0 & Medium \\
\hline Suprabha, 2013, [145] & $11-13$ & India & 857 & 59.4 & High \\
\hline Panagidis, 2012, [146] & 12 & Germany & 951 & 32.6 & High \\
\hline Shailee-1, 2012, [147] & 12 & India & 1011 & 32.6 & Medium \\
\hline Shailee-2, 2012, [147] & 15 & India & 1011 & 42.2 & High \\
\hline Lagana, 2012, [148] & $7-15$ & Albanian & 2617 & 88.9 & Medium \\
\hline Subedi, 2011, [148] & $12-13$ & Nepal & 325 & 53.2 & Medium \\
\hline Shekar, 2011, [149] & $12-15$ & India & 474 & 56.3 & High \\
\hline Oulis-1, 2010, [150] & 12 & Greece & 1224 & 80.0 & High \\
\hline Oulis-2, 2010, [150] & 15 & Greece & 1257 & 83.0 & High \\
\hline Jamelli, 2010, [151] & 12 & Brazil & 689 & 71.8 & High \\
\hline Kanagaratnam, 2009, [152] & 9 & New Zealand & 612 & 54.9 & High \\
\hline Bissar, 2008, [153] & $11-14$ & Germany & 570 & 42.3 & High \\
\hline Ferro, 2007, [154] & 12 & Italy & 260 & 56.9 & High \\
\hline Moreira, 2006, [155] & $12-15$ & Brazil & 1665 & 50.9 & High \\
\hline Schulte, 2004, [156] & 12 & Germany & 43,950 & 39.3 & High \\
\hline Paredes, 2005, [157] & $6-10$ & Spain & 600 & 47.2 & High \\
\hline Mestriner, 2005, [158] & 12 & Brazil & 256 & 53.9 & High \\
\hline Traebert, 2002, [159] & 12 & Brazil & 803 & 62.1 & High \\
\hline
\end{tabular}




\section{Funnel Plot of Standard Error by Logit event rate}

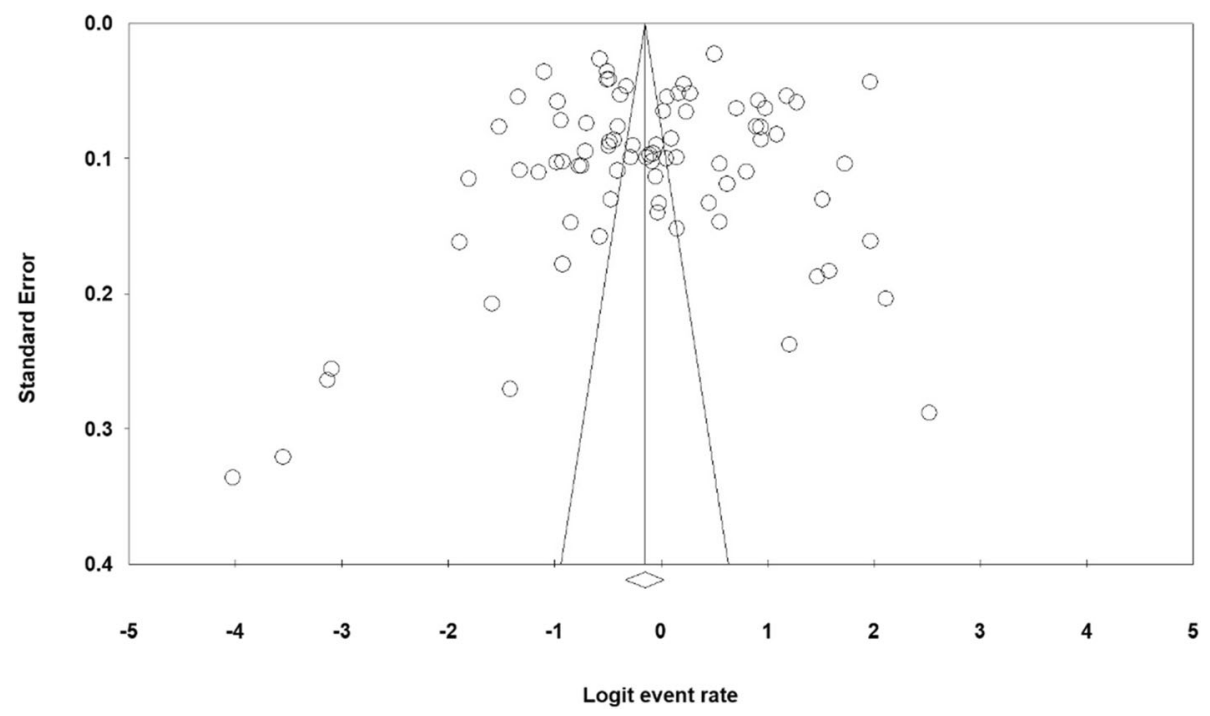

Fig. 2 Funnel plot of the results of the prevalence of dental caries in primary teeth in children

Cumulative risk of each study's addition to the metaanalysis are reported in Figs. 11 and 12.

\section{Discussion}

In this study, the prevalence of dental caries in studies conducted throughout the world was investigated, and it was reported that the overall prevalence of dental caries in primary teeth in children was $46.2 \%$. Early childhood caries (ECC) in developing countries was reported to be more than in developed countries [1]. Also, in the present study, the overall prevalence of dental caries in permanent teeth in children was $53.8 \%$.

Differences in the prevalence of dental caries in developed and underdeveloped countries may be due to differences in the age groups studied, but may also be due to ethnic, cultural, geographic, racial, and developmental differences as well as access to dental services, behavioral habits, health care behaviors,

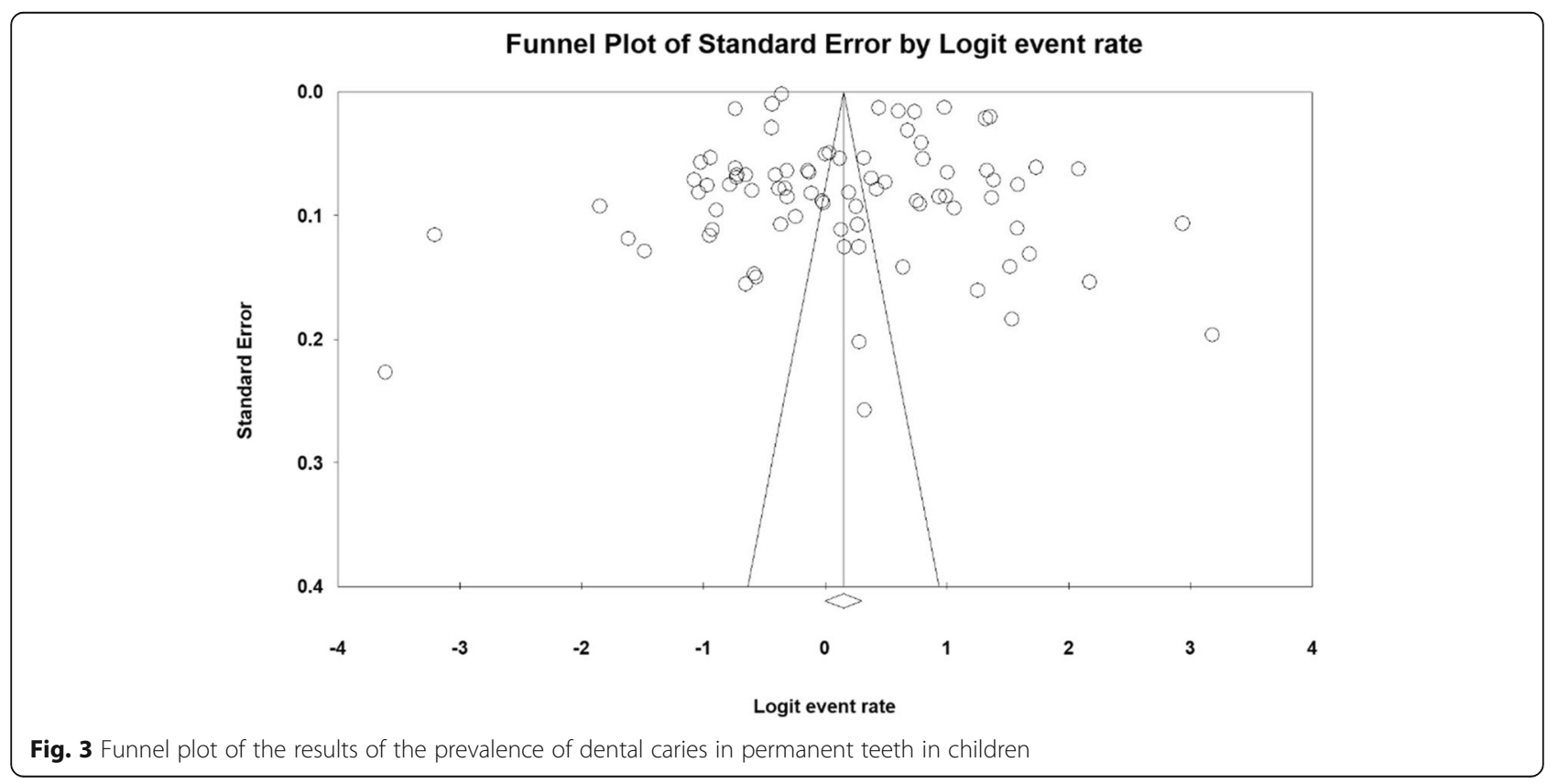




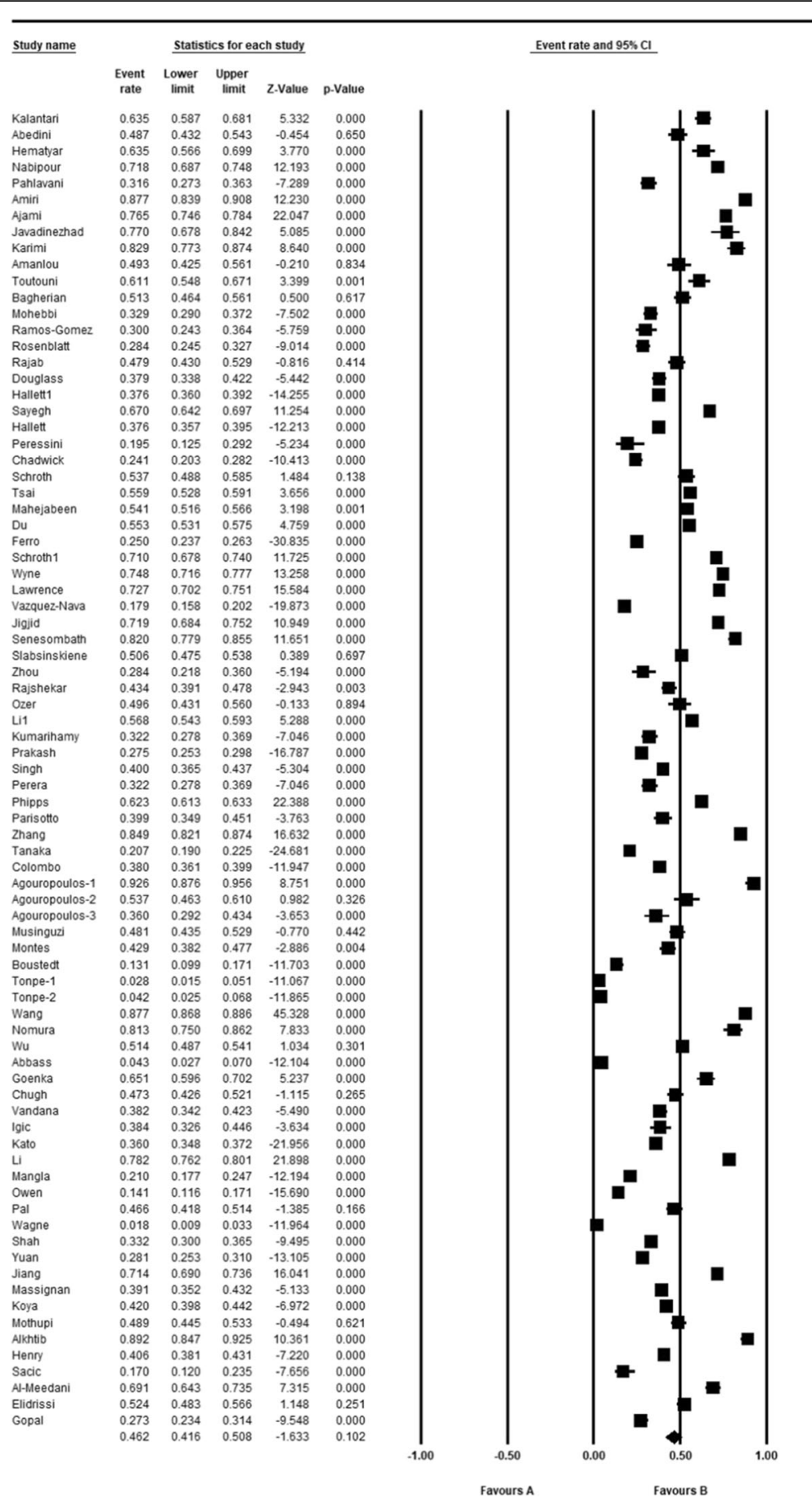

\section{Meta Analysis}

Fig. 4 Forest plot of the results of Prevalence of dental caries in primary teeth and 95\% confidence intervals worldwide 


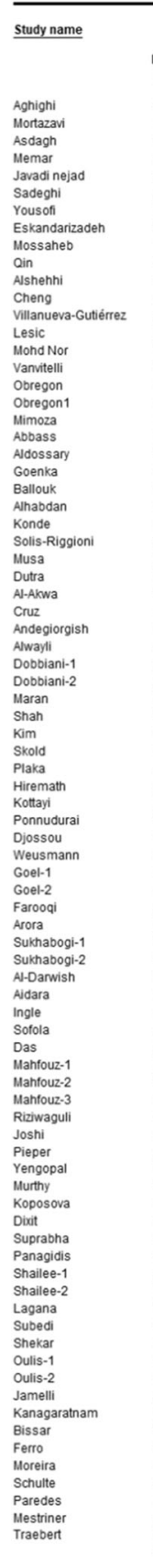

\begin{tabular}{cccc}
\multicolumn{5}{c}{ Statistics for each study } \\
$\begin{array}{c}\text { Event } \\
\text { rate }\end{array} \begin{array}{c}\text { Lower } \\
\text { limit }\end{array} \begin{array}{c}\text { Upper } \\
\text { limit }\end{array} \quad$ Z-Value & $p$-Value
\end{tabular}

$\begin{array}{lllll}0.663 & 0.649 & 0.677 & 21.861 & 0.000\end{array}$

$\begin{array}{rrrrr}0.655 & 0.589 & 0.714 & 4.507 & 0.000 \\ 0.797 & 0.769 & 0.823 & 16.007 & 0.000\end{array}$

\begin{tabular}{llllll}
0.797 & 0.768 & 0.823 & 4607 & 0.000 \\
\hline & 0.843 & 0.806 & 0.874 & 12807 & 0.000
\end{tabular}

$\begin{array}{llllll}0.821 & 0.776 & 0.858 & 10.756 & 0.000\end{array}$

$\begin{array}{llllll}0.686 & 0.646 & 0.723 & 8.589 & 0.000\end{array}$

$\begin{array}{lllll}0.898 & 0.867 & 0.922 & 14.118 & 0.000\end{array}$

$\begin{array}{rrrrr}0.795 & 0.789 & 0.801 & 67.827 & 0.000 \\ 0.823 & 0.764 & 0.869 & 8.351 & 0.000\end{array}$

$\begin{array}{rrrrr}0.823 & 0.764 & 0.869 & 8.351 & 0.000 \\ 0.392 & 0.379 & 0.405 & -15.247 & 0.000\end{array}$

$\begin{array}{llllll}0.581 & 0.455 & 0.696 & 1.264 & 0.206\end{array}$

$\begin{array}{llllll}0.411 & 0.411 & 0.412 & -192543 & 0.000\end{array}$

$\begin{array}{lllll}0.354 & 0.319 & 0.391 & -7.522 & 0.000\end{array}$

$\begin{array}{rrrrr}0.500 & 0.476 & 0.525 & 0.025 & 0.980 \\ 0.743 & 0.706 & 0.776 & 11310 & 0.000\end{array}$

$\begin{array}{rrrrr}0.743 & 0.706 & 0.776 & 11.310 & 0.000 \\ 0.291 & 0.253 & 0.331 & -9330 & 0.000\end{array}$

$\begin{array}{rrrrr}0.291 & 0.253 & 0.331 & -9.330 & 0.000 \\ 0.255 & 0.229 & 0.282 & -15.131 & 0.000\end{array}$

$\begin{array}{lllll}0.255 & 0.229 & 0.282 & -15.131 & 0.000 \\ 0.262 & 0.232 & 0.294 & -12.751 & 0.000\end{array}$

$\begin{array}{rrrrr}0.262 & 0.232 & 0.294 & -12.751 & 0.000 \\ 0.284 & 0.242 & 0.330 & -8.322 & 0.000\end{array}$

$\begin{array}{lllllll}0.950 & 0.939 & 0.959 & 27.1584 & 0.000\end{array}$

$\begin{array}{llllll}0.567 & 0.514 & 0.617 & 2.494 & 0.013\end{array}$

$\begin{array}{llllll}0.791 & 0.769 & 0.811 & 20.940 & 0.000\end{array}$

$\begin{array}{llllll}0.829 & 0.796 & 0.857 & 14.280 & 0.000\end{array}$

$\begin{array}{lllll}0.136 & 0.116 & 0.159 & -20.042 & 0.000\end{array}$

$\begin{array}{llllll}0.358 & 0.295 & 0.427 & -3.964 & 0.000\end{array}$

$\begin{array}{llllll}0.324 & 0.318 & 0.330 & -39.095 & 0.000\end{array}$

$\begin{array}{llllll}0.676 & 0.569 & 0.683 & 45.648 & 0.0000\end{array}$

$\begin{array}{llllll}0.342 & 0.277 & 0.414 & -4201 & 0.000\end{array}$

$\begin{array}{lllll}0.778 & 0.719 & 0.827 & 7.812 & 0.000\end{array}$

$\begin{array}{lllll}0.646 & 0.639 & 0.653 & 38.466 & 0.000\end{array}$

$\begin{array}{lllll}0.440 & 0.392 & 0.489 & -2.394 & 0.017\end{array}$

$\begin{array}{lllll}0.185 & 0.150 & 0.226 & -11.516 & 0.000 \\ 0.732 & 0.0706 & 0.750 & 15420 & 0000\end{array}$

$\begin{array}{rrrrr}0.732 & 0.706 & 0.756 & 15.426 & 0.000 \\ 0.314 & 0.283 & 0.346 & -10.463 & 0.000\end{array}$

$\begin{array}{rrrrr}0.344 & 0.283 & 0.346 & -10.463 & 0.000 \\ 0.494 & 0.451 & 0.537 & -0.265 & 0.791\end{array}$

$\begin{array}{llllll}0.026 & 0.017 & 0.041 & -15922 & 0.000\end{array}$

$\begin{array}{lllll}0.363 & 0.298 & 0.433 & -3.765 & 0.000\end{array}$

$\begin{array}{lllll}0.789 & 0.782 & 0.796 & 61.830 & 0.000 \\ 0.039 & 0.031 & 0.040 & -27.743 & 0.000\end{array}$

$\begin{array}{lllll}0.039 & 0.031 & 0.048 & -27.743 & 0.000 \\ 0.688 & 0.671 & 0.705 & 19385 & 0.000\end{array}$

$\begin{array}{lllll}0.688 & 0.671 & 0.705 & 19.385 & 0.000 \\ 0.497 & 0.453 & 0.541 & -0.135 & 0.893\end{array}$

$\begin{array}{lllll}0.497 & 0.453 & 0.541 & -0.135 & 0.893 \\ 0.609 & 0.603 & 0.615 & 34.200 & 0.000\end{array}$

$\begin{array}{lllll}0.343 & 0.314 & 0.373 & -9.733 & 0.000\end{array}$

$\begin{array}{llllll}0.465 & 0.434 & 0.496 & -9.7221 & 0.000 \\ 0.026\end{array}$

$\begin{array}{llllll}0.730 & 0.696 & 0.761 & 11.772 & 0.000\end{array}$

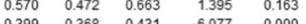

$\begin{array}{lllll}0.399 & 0.368 & 0.431 & -6.077 & 0.000 \\ . .467 & 0.435 & 0.499 & -2.041 & 0.041\end{array}$

$\begin{array}{llllll}0.850 & 0.834 & 0.865 & 28.470 & 0.000\end{array}$

$\begin{array}{llllll}0.960 & 0.942 & 0.973 & 16.197 & 0.000\end{array}$

$\begin{array}{lllll}0.530 & 0.504 & 0.556 & 2244 & 0.025\end{array}$

$\begin{array}{lllllll}0 & 031 & 0.650 & 0.302 & -17765 & 0.000\end{array}$

$\begin{array}{llllll}0.406 & 0.370 & 0.444 & -4.852 & 0.000\end{array}$

$\begin{array}{lllll}0.418 & 0.381 & 0.456 & -4247 & 0.000\end{array}$

$\begin{array}{lllll}0.604 & 0.567 & 0.640 & 5.379 & 0.000\end{array}$

$\begin{array}{lllll}0.265 & 0.244 & 0.287 & -18.009 & 0.000\end{array}$

$\begin{array}{llllll}0.691 & 0.668 & 0.713 & 14.848 & 0.000\end{array}$

$\begin{array}{llllll}0.727 & 0.722 & 0.732 & 76.761 & 0.000\end{array}$

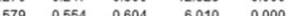

\begin{tabular}{llllll}
\hline 680 & 0.554 & 0.04 & -010 & 0.000
\end{tabular}

$\begin{array}{lllll}0.410 & 0.360 & 0.461 & -3.402 & 0.001\end{array}$

$\begin{array}{rrrrr}0.594 & 0.561 & 0.626 & 5.467 & 0.000\end{array}$

$\begin{array}{lllll}0.326 & 0.297 & 0.356 & -10.501 & 0.000 \\ & 0.356 & -10801 & 0.000\end{array}$

$\begin{array}{lllll}0.326 & 0.298 & 0.356 & -10.801 & 0.000 \\ 0.422 & 0.392 & 0.453 & -4918 & 0.000\end{array}$

$\begin{array}{lllll}0.422 & 0.392 & 0.453 & -4.918 & 0.000 \\ 0.889 & 0.877 & 0.901 & 33.440 & 0.000\end{array}$

$\begin{array}{rrrrr}0.889 & 0.877 & 0.901 & 33.440 & 0.000 \\ 0.532 & 0.478 & 0.586 & 1.164 & 0.244\end{array}$

$\begin{array}{llllll}0.563 & 0.518 & 0.607 & 2.748 & 0.006\end{array}$

$\begin{array}{lllll}0.800 & 0.776 & 0.821 & 19.392 & 0.000\end{array}$

$\begin{array}{lllll}0.830 & 0.808 & 0.850 & 21.106 & 0.000\end{array}$

$\begin{array}{lllll}0.718 & 0.684 & 0.751 & 11.058 & 0.000\end{array}$

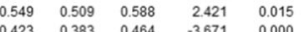

$\begin{array}{lllll}0.423 & 0.383 & 0.464 & -3.671 & 0.000 \\ 0.569 & 0.508 & 0.628 & 2225 & 0.026\end{array}$

$\begin{array}{lllll}0.569 & 0.508 & 0.628 & 2.225 & 0.026 \\ 0.509 & 0.485 & 0.533 & 0.711 & 0.477\end{array}$

$\begin{array}{lllll}0.393 & 0.388 & 0.398 & -44.515 & 0.000\end{array}$

$\begin{array}{lllll}0.472 & 0.432 & 0.512 & -1.387 & 0.165 \\ 0.539 & 0.470 & 0.599 & 1249 & 0.212\end{array}$

$\begin{array}{lllll}0.539 & 0.478 & 0.599 & 1.249 & 0.212 \\ 0.621 & 0.587 & 0.654 & 6.811 & 0.000\end{array}$

$\begin{array}{llllll}0.538 & 0.500 & 0.575 & 1.951 & 0.051\end{array}$
Event rate and $95 \% \mathrm{Cl}$

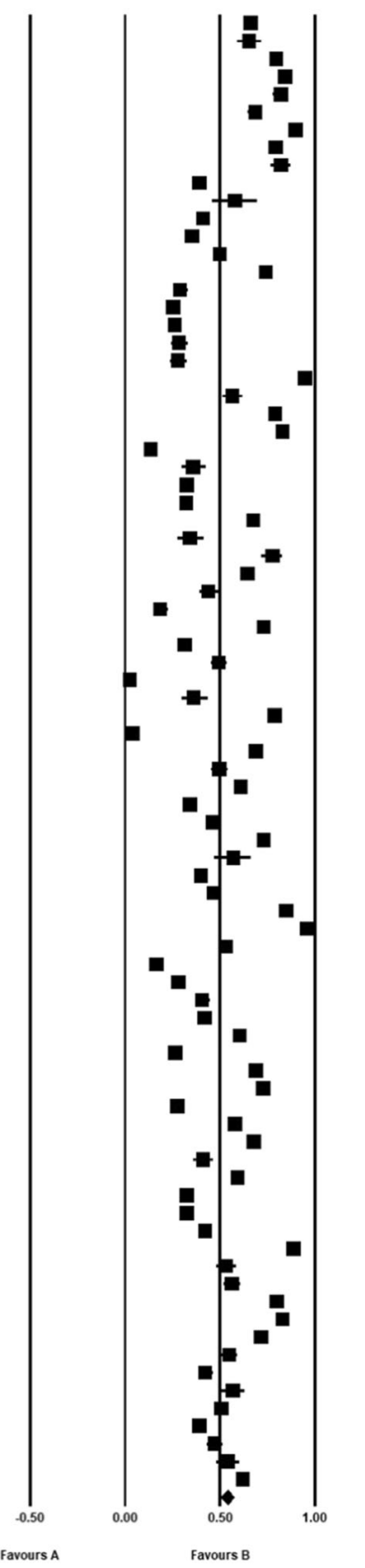

\section{Meta Analysis}

Fig. 5 Forest plot of the results of Prevalence of dental caries in permanent teeth and $95 \%$ confidence intervals worldwide

nutritional habits and behaviors, and lifestyle [144]. The effects of parents' lack of awareness of their children's tooth decay status as well as neglect and attention discrimination can also be well documented in the study of Nag et al. [160], suggesting that in the age group of 6 to 18 years, caries rates were higher in girls than in boys, as girls are more neglected by parents than boys. Although there has been no difference 


\section{Regression of Sample on Logit event rate}

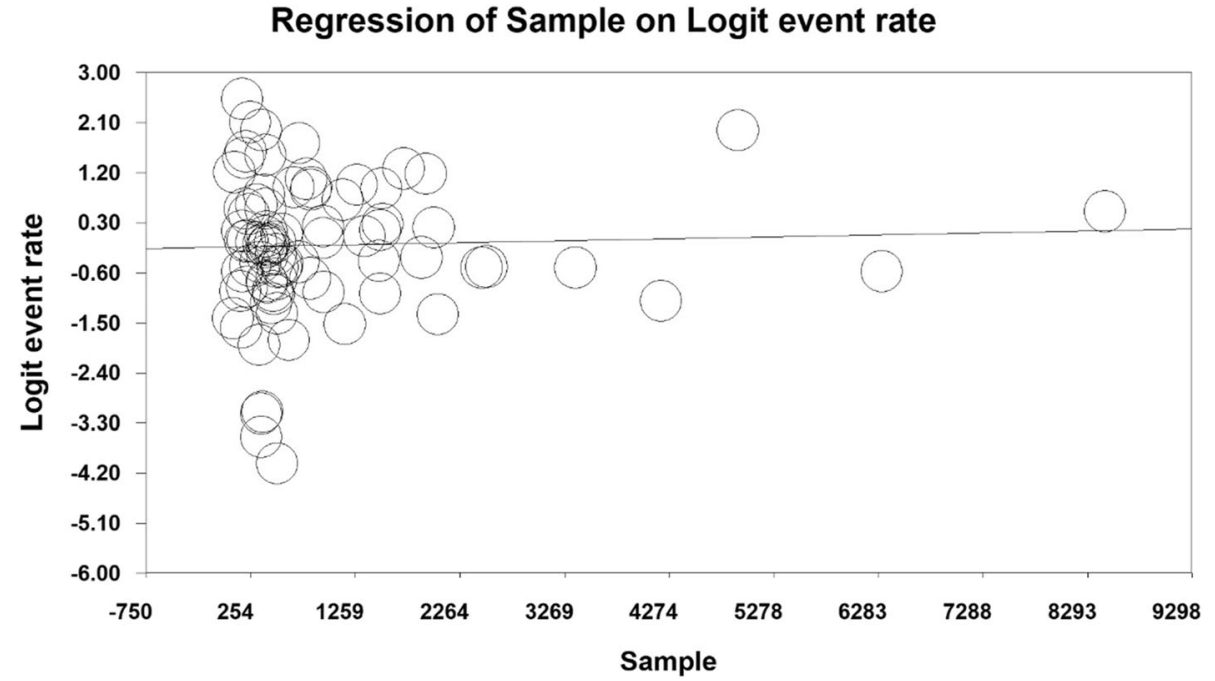

Fig. 6 Meta-regression analysis of the relationship between the sample size and the prevalence of dental caries in primary teeth in children in the world

in the prevalence rate of both sexes in the current systematic study, terms of access to health services and lack of parental awareness of and attention to children's dental caries are known as the most important factors in its development [2], so discrimination and inequality in the upbringing of children in the family can also multiply the impact of such a situation.

In the present meta-analysis study, the prevalence of dental caries in primary and permanent teeth in different continents is presented in Table 3 and Fig. 10, which was higher in the African continent and secondarily in the Asian continent.

According to the World Health Organization's Healthy People Plan, 90\% of children between the ages of 5 and 6 should be free of tooth decay by 2010. However, according to the findings of this study which were based on the reviews performed in the studies searched, the prevalence of dental caries in children in most countries was found to be very high. Such a situation gives rise to worrying conditions in terms of tooth decay in adulthood and will also

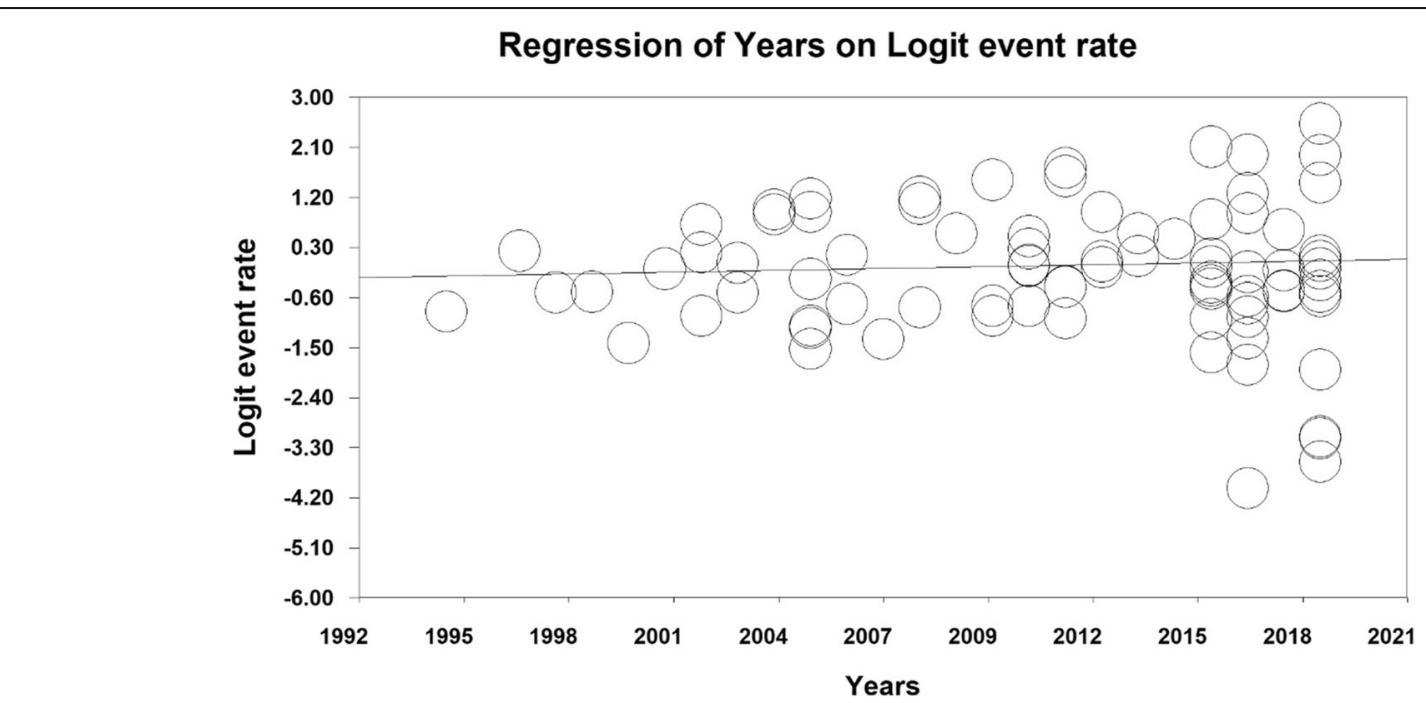

Fig. 7 Meta-regression analysis of the relationship between the year of study and the prevalence of dental caries in primary teeth in children in the world 


\section{Regression of Sample on Logit event rate}

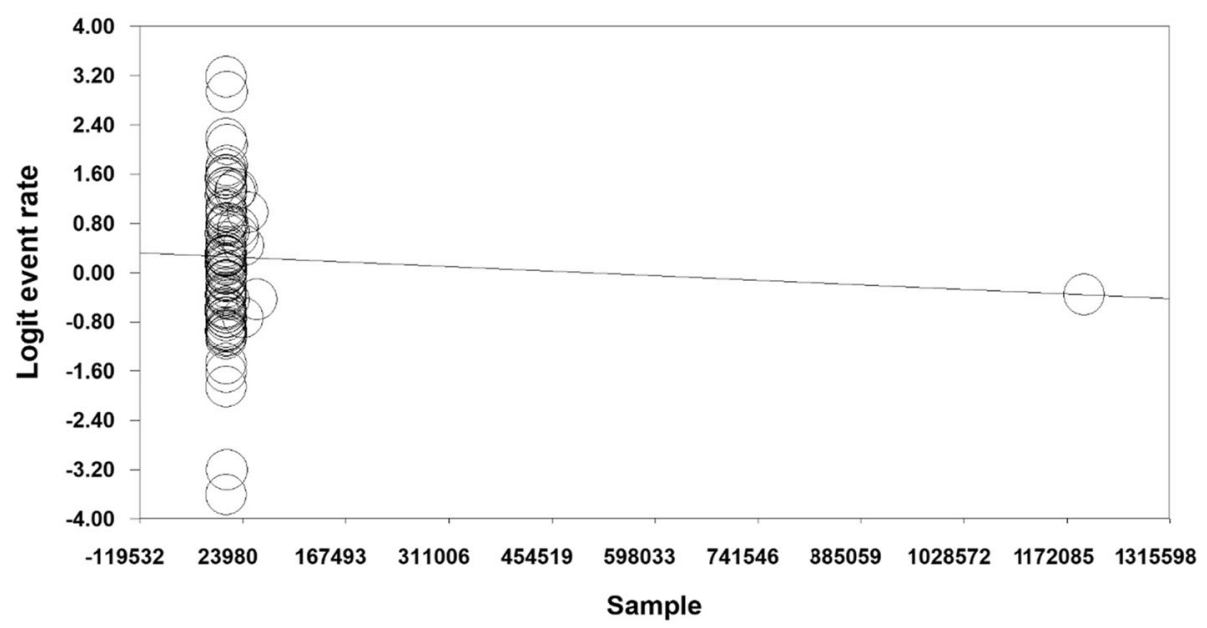

Fig. 8 Meta-regression analysis of the relationship between the sample size and the prevalence of dental caries in permanent teeth in children in the world

impose enormous tooth repair costs on the country's health sector. Such a situation in the country, in addition to what has been said, as well as conditions such as inequality in access to health care services, inequality in developmental and economic situation in different countries and different parts of countries may indicate that the lack of awareness of the health and preservation of primary teeth in all families with different socioeconomic status has been considered a serious problem [3] and a barrier to the provision of preventive and health services.
Families and parents should know that child dental care must start from the mother's pregnancy; children born to mothers with multiple dental caries are more likely to develop caries in the later stages of their lives. Cariogenic bacteria are usually transferred through the use of a spoon or a bottle of milk from the mother's moth to the child's mouth for the first time, so breast feeding should be avoided as much as possible during the baby's sleep. Regular dental appointments should be provided from the beginning of the baby's primary teeth eruption, especially with the eruption of the first

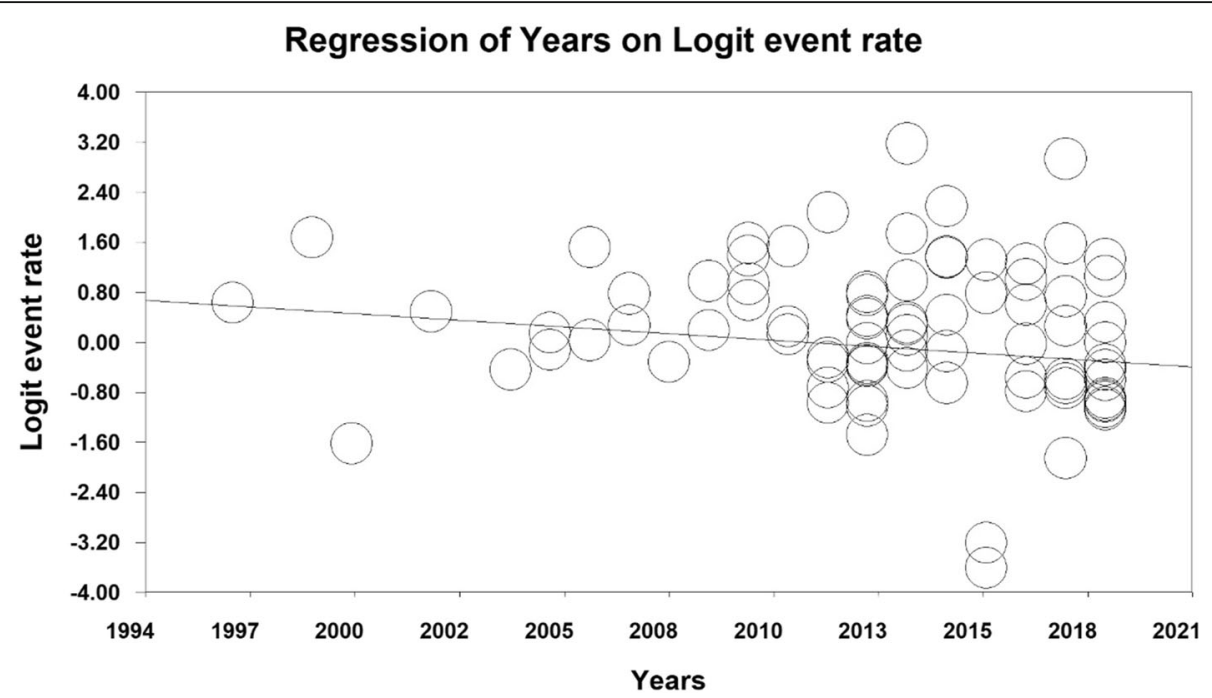

Fig. 9 Meta-regression analysis of the relationship between the year of study and the prevalence of dental caries in permanent teeth in children in the world 
Table 3 Investigating the prevalence of dental caries in primary and permanent teeth in children in different continents

\begin{tabular}{lllllll}
\hline Tooth type & continents & Number of articles & Sample Size & $\mathbf{I}^{\mathbf{2}}$ & Egger Test & Prevalence \% \\
\hline Caries in primary teeth & Asia & 50 & 54,680 & 99.3 & 0.756 & $52.6(95 \% \mathrm{Cl}: 46.7-58.5)$ \\
& Europe & 10 & 9977 & 98.4 & 0.152 & $21.4(95 \% \mathrm{Cl}: 15.3-29.1)$ \\
& America & 14 & 6825 & 98.7 & 0.742 & $45.8(95 \% \mathrm{Cl}: 34.2-58)$ \\
& Africa & 5 & 3004 & 95.5 & 0.220 & $53.1(95 \% \mathrm{Cl}: 44.3-61.7)$ \\
& Australia & 3 & 6472 & 98.3 & 0.296 & $28.5(95 \% \mathrm{Cl}: 20.3-38.5)$ \\
Permanent dental caries & Asia & 50 & $1,334,133$ & 99.8 & 0.284 & $58.8(95 \% \mathrm{Cl}: 53.4-64)$ \\
& Europe & 22 & 115,141 & 99.8 & 0.175 & $44.1(95 \% \mathrm{Cl}: 36.1-52.5)$ \\
& America & 7 & 5009 & 98.2 & 0.763 & $48.9(95 \% \mathrm{Cl}: 37.6-60.3)$ \\
& Africa & 5 & 2794 & 99.3 & 0.220 & $58.9(95 \% \mathrm{Cl}: 29.4-83.1)$ \\
& Australia & 1 & 612 & - & - & $54.9(95 \% \mathrm{Cl}: 50.9-58.8)$ \\
\hline
\end{tabular}

permanent tooth, first molar tooth or the 6the tooth. The tooth develops immediately after the last primary tooth at the age of six and is most likely to be decayed. The American Academy of Pediatric Dentistry, the American Dental Association, and the American Academy of General Dentistry all recommend making sure to see a dentist 6 months after the eruption of teeth in children, before the age of one year's [161-166]. One of the most important and available measures to prevent caries, especially primary teeth in children, is performing dental procedures such as fissure sealant and fluoride therapy. In the fissure sealant method, deep grooves in the surface of the tooth are covered with a thin layer of toothcolored material, thereby preventing the spread of cariogenic bacteria in tooth grooves. Other methods are caries prevention [167] and[168].

Given the high prevalence of primary and permanent dental caries in children worldwide, it is recommended that providing educational programs and interventions in primary and permanent dental health care especially for mothers, nurses, and child educators be of special interest to health services policy-makers and providers. Planning to provide educational programs and inexpensive dental and oral health services as well as ease of access to such services for children by the health system of countries is noted as well.

\section{Strength and limitation}

The most important strength of this study is that it has been studied for the first time in the world, includes all data sources and high-quality studies, and also analysis based on different continents for the use of the World Health Organization. The most important limitation of the present study is inaccessibility to the full text of the articles, incomplete search, and poor quality of some studies, as well as restricted search based on Persian and English languages.

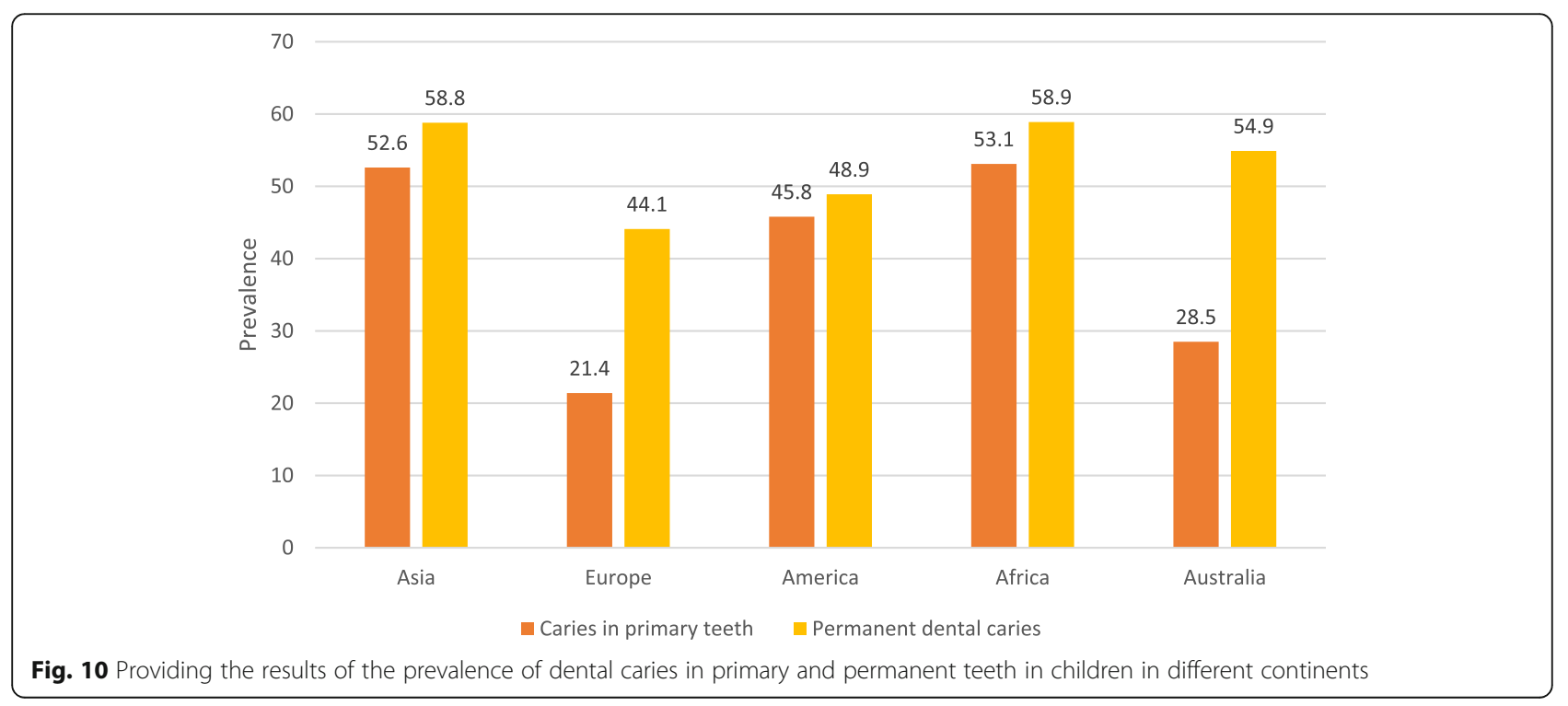




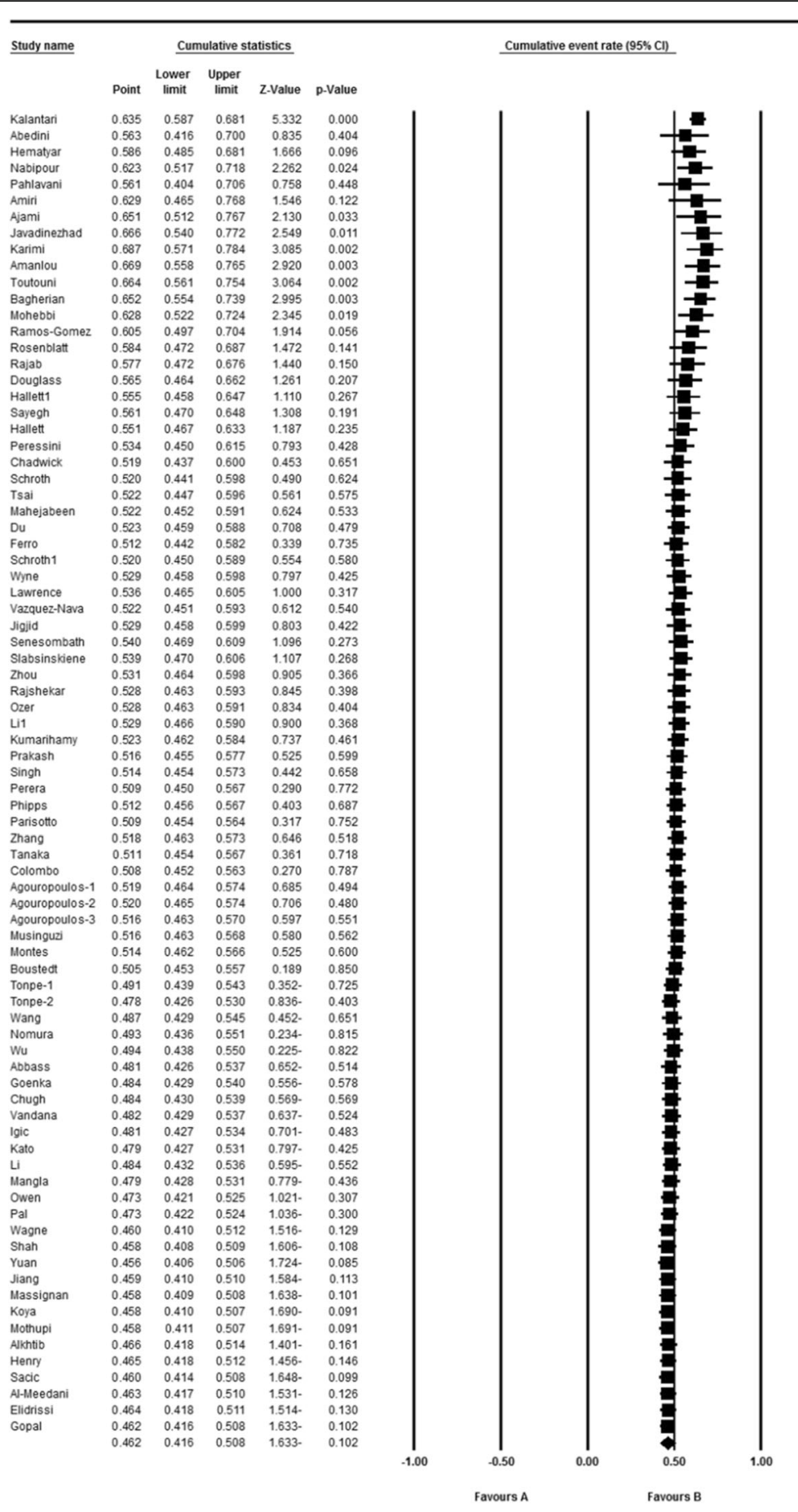

\section{Meta Analysis}

Fig. 11 Result of cumulative meta-analysis based on primary dental caries 


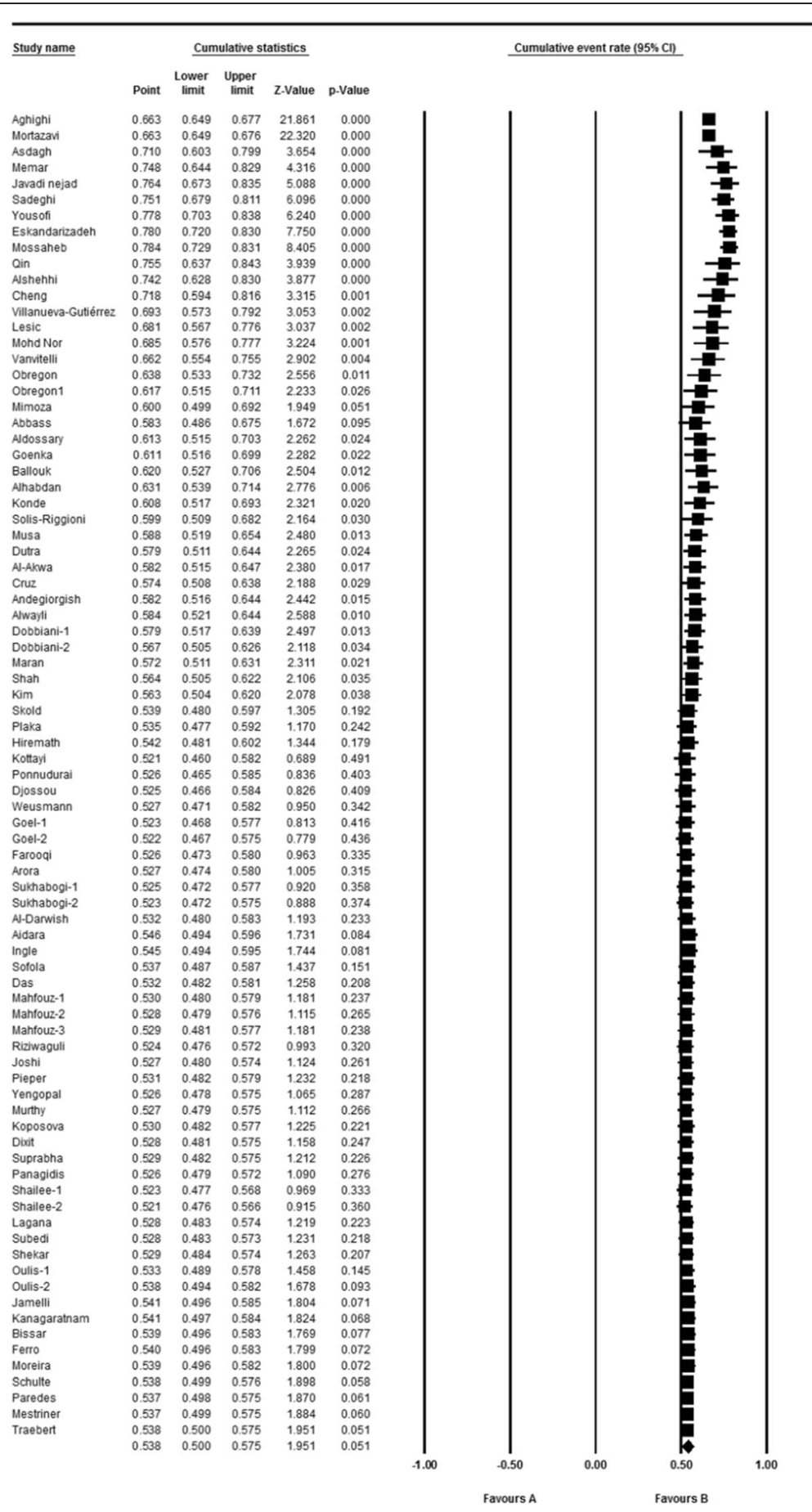

\section{Meta Analysis}

Fig. 12 Result of cumulative meta-analysis based on permanent dental caries 


\section{Conclusion}

The results of this study showed that the prevalence of dental caries in primary and permanent teeth in children in the world was found to be high. Therefore, appropriate strategies should be implemented to improve the aforementioned situation and to troubleshoot and monitor at all levels by providing feedback to hospitals. Also, the prevalence of dental caries in primary and permanent teeth in children of Africa is higher than other continents and requires special attention of the World Health Organization to this continent in improving the oral health of children. These strategies can include providing educational programs to parents, periodic dental care for children, and fluoride therapy in childhood for the African continent.

\section{Abbreviations}

ECC: Early Childhood Caries; WHO: World Health Organization; DMF: DecayMissing-Filled; SID: Scientific Information Database; MESH: Medical Subject Headings; ISI: Web of Science; PRISMA: Preferred Reporting Items for Systematic Reviews and Meta-Analysis; STROBE: Strengthening the Reporting of Observational Studies in Epidemiology for cross- sectional Study

\section{Acknowledgements}

We hereby express our gratitude and appreciation to the respected authorities of that center for bearing the financial costs of this study.

\section{Authors' contributions}

MK and AA contributed to the design, MM and RJ statistical analysis, participated in most of the study steps. AVR and SHSH prepared the manuscript. NS and MM assisted in designing the study, and helped in the, interpretation of the study. All authors have read and approved the content of the manuscript.

\section{Funding}

By Student Research Committee of Kermanshah University of Medical Sciences, Deputy for Research and Technology, Kermanshah University of Medical Sciences (IR) (3009346).

\section{Availability of data and materials}

Datasets are available through the corresponding author upon reasonable request.

\section{Ethics approval and consent to participate}

Not declared.

\section{Consent for publication}

Not applicable.

\section{Competing interests}

The authors declare that they have no conflict of interest.

\section{Author details}

${ }^{1}$ Department of Nursing, School of Nursing and Midwifery, Kermanshah University of Medical Sciences, Kermanshah, Iran. ${ }^{2}$ Department of Biology, Faculty of Science, University Putra Malaysia, Serdang, Selangor, Malaysia. ${ }^{3}$ Department of Biostatistics, School of Health, Kermanshah University of Medical Sciences, Kermanshah, Iran.
Received: 20 April 2020 Accepted: 17 September 2020

Published online: 06 October 2020

\section{References}

1. Nematollahi $H$, Mehrabkhani M, Sheykhani M. Assessing the relationship between diet and prevalence of early childhood caries in Birjand preschool children. J Dent. 2007:8(1):70-85

2. Wagle M, D'Antonio F, Reierth E, Basnet P, Trovik TA, Orsini G, Manzoli L, Acharya G. Dental caries and preterm birth: a systematic review and metaanalysis. BMJ Open. 2018;8(3):e018556.

3. Broumand S, Sharififar S, Alikhani S. The study of caries free indicator of milk teeth in children age 3-6 at dare care center affiliated to health centers of Army; 2006.

4. Moynihan P, Petersen PE. Diet, nutrition and the prevention of dental diseases. Public Health Nutr. 2004;7(1A):201-26.

5. Hallett KB, O'Rourke PK. Pattern and severity of early childhood caries. Community Dent Oral Epidemiol. 2006:34(1):25-35.

6. McDonald RE, Avery DR. Dentistry for the child and adolescent, Mosby Incorporated; 2004.

7. Bayat Movahed S, Samadzadeh H, Ziyarati L, Memory N, Khosravi R, Eshkevari PS. Oral health of Iranian children in 2004: a national pathfinder survey of dental caries and treatment needs; 2011.

8. World Health Organization. Oral health surveys-basic methods. 4th ed. Geneva: WHO; 1997.

9. Vanobbergen J, Lesaffre E, Garcia-Zattera M, Jara A, Martens L, Declerck D. Caries patterns in primary dentition in 3-, 5-and 7-year-old children: spatial correlation and preventive consequences. Caries Res. 2007;41(1):16-25.

10. Vandenbroucke JP, VON Elm E, Altman DG, Gotzsche PC, Mulrow CD, Pocock SJ, et al. Strengthening the reporting of observational studies in epidemiology (STROBE): explantion and elaboration. Epidemiology. 2007; 18(6):805-35.

11. Kalantari B, Rahmannia J, Hatami H, Karkhaneh S, Farsar A, Sharifpoor A, Zahedi B. The prevalence of dental caries in primary molars and its related factors in 6 and 7 years old children in Shemiranat health center. J Health Field. 2014;1(4):7-13.

12. Abedini $H$. Prevalence and causes of decay in primary teeth of children aged 2-6 years in Kashan; 2013.

13. Hematyar M, Masnavi A. Prevalence and risk factors of dental decays in 3-7 years old children referred to pediatric clinics of Islamic Azad University. J Qazvin Univ Med Sci. 2009;13(3):87-94.

14. Nabipour AR, Azvar K, Zolala F, Ahmadinia H, Soltani Z. The prevalence of early dental caries and its contributing factors among 3-6-year-old children in Varamin/Iran. Health Dev J. 2013;2(1):12-0.

15. Pahlavani Z, Egjbalian F, Moksefesfahani F, Chitgar Z. Determination of frequency and pattern of dental caries and its effective factors in 2 to 6 year old children in Hamedan. Feyz Sci J. 2008;12(1):81-7 [In Persian].

16. Amiri, S., M. Veissi, M. Saleki, M. Rahmani And M. Haghighizadeh. The relationship between dental caries with dietary habits and body mass index in 4-6 years old kindergartens in Ahvaz. 2017.

17. Ajami B, Ebrahimi M. Evaluation of oral health status amongst 6-7-year-old children in Mashhad in 2001. J Mashhad Dental Sch. 2005;29(Issue):235-42.

18. Javadinezhad SH, Karami M, Fayz E. Nutritional habits and the prevalence of dental caries in preterm or low birth weight children. J Shiraz Dent. 2008; 9(3):291-8 [In Persian]

19. Karimi Shahanjarini A, Makvandi Z, Faradmal J, Bashirian S, Hazavehei M. Assessing the tooth decay status of 2-5 years children and the role of their mothers' caring behaviors. Sci J Hamadan Nurs Midwifery Faculty. 2012; 21(4):41-50 [In Persian].

20. Amanlou M, Jafari S, Afzalianmand N, Omrany ZB, Farsam H, Nabati F, Bagherzadeh K. Association of Saliva Fluoride level and socioeconomic factors with dental caries in 3-6 years old children in Tehran-Iran. Iran J Pharm Res. 2011;10(1):159.

21. Toutouni $H$, Nokhostin M-R, Amaechi BT, Zafarmand $A H$. The prevalence of early childhood caries among 24 to 36 months old children of Iran: using the novel ICDAS-II method. J Dent. 2015;16(4):362.

22. Bagherian A, Sadeghi M. Association between dental caries and age-specific body mass index in preschool children of an Iranian population. Indian J Dent Res. 2013;24(1):66.

23. Mohebbi SZ, Virtanen Jl, Vahid-Golpayegani M, Vehkalahti MM. Early childhood caries and dental plaque among 1-3-year-olds in Tehran, Iran. J Indian Soc Pedod Prev Dent. 2006;24(4):177. 
24. Ramos-Gomez FJ, Tomar SL, Ellison J, Artiga N, Sintes J, Vicuna G. Assessment of early childhood caries and dietary habits in a population of migrant Hispanic children in Stockton, California. ASDC J Dent Child. 1999; 66(6):395-403, 366

25. Rosenblatt A, Zarzar P. The prevalence of early childhood caries in 12-to 36month-old children in Recife, Brazil. J Dent Child. 2002;69(3):319-24.

26. Rajab LD, Hamdan M. Early childhood caries and risk factors in Jordan. Community Dent Health. 2002;19(4):224-9.

27. Douglass J, Montero M, Thibodeau E, Mathieu G. Dental caries experience in a Connecticut head start program in 1991 and 1999. Pediatr Dent. 2002;24(4):309-14.

28. Hallett KB, O'Rourke PK. Early childhood caries and infant feeding practice. Community Dent Health. 2002;19(4):237-42.

29. Sayegh A, Dini E, Holt R, Bedi R. Caries in preschool children in Amman, Jordan and the relationship to socio-demographic factors. Int Dent J. 2002; 52(2):87-93.

30. Hallett K, O'Rourke P. Social and behavioural determinants of early childhood caries. Aust Dent J. 2003:48(1):27-33.

31. Peressini S, Leake JL, Mayhall JT, Maar M, Trudeau R. Prevalence of early childhood caries among first nations children, district of Manitoulin, Ontario. Int J Paediatr Dent. 2004;14(2):101-10.

32. Chadwick BL, Treasure ET, Playle RA. A randomised controlled trial to determine the effectiveness of glass ionomer sealants in pre-school children. Caries Res. 2005;39(1):34-40.

33. Schroth RJ, Pang JL, Levi JA, Martens PJ, Brownell MD. Trends in pediatric dental surgery for severe early childhood caries in Manitoba, Canada. J Can Dent Assoc. 2014;80:e65.

34. Tsai Al, Chen CY, Li LA, Hsiang CL, Hsu KH. Risk indicators for early childhood caries in Taiwan. Community Dent Oral Epidemiol. 2006;34(6): 437-45.

35. Mahejabeen R, Sudha P, Kulkarni S, Anegundi R. Dental caries prevalence among preschool children of Hubli: Dharwad city. J Indian Soc Pedod Prev Dent. 2006;24(1):19.

36. Du M, Luo Y, Zeng X, Alkhatib N, Bedi R. Caries in preschool children and its risk factors in 2 provinces in China. Quintessence Int. 2007;38(2):143-51.

37. Ferro R, Besostri A, Olivieri A, Stellini E, Mazzoleni S. Preschoolers' dental caries experience in and its trend over 20 years in a north-east Italian health district. Eur J Paediatr Dent. 2007;8(4):199.

38. Schroth RJ, Cheba V. Determining the prevalence and risk factors for early childhood caries in a community dental health clinic. Pediatr Dent. 2007;29(5):38796.

39. Wyne A. Caries prevalence, severity, and pattern in preschool children. J Contemp Dent Pract. 2008;9(3):24-31.

40. Lawrence HP, Binguis D, Douglas J, McKeown L, Switzer B, Figueiredo R, Laporte A. A 2-year community-randomized controlled trial of fluoride varnish to prevent early childhood caries in Aboriginal children. Community Dent Oral Epidemiol. 2008;36(6):503-16.

41. Vázquez-Nava F, Vázquez R, Saldivar G, Beltrán G, Almeida A, Vázquez R. Allergic rhinitis, feeding and oral habits, toothbrushing and socioeconomic status. Caries Res. 2008;42(2):141-7.

42. Jigjid B, Ueno M, Shinada K, Kawaguchi Y. Early childhood caries and related risk factors in Mongolian children. Community Dent Health. 2009;26(2):121.

43. Senesombath S, Nakornchai S, Banditsing P, Lexomboon D. Early childhood caries and related factors in Vientiane, Lao PDR; 2010.

44. Slabšinskienè E, Milčiuvienè $S$, Narbutaitè J, Vasiliauskienè I, Andruškevičienè V, Bendoraitienè E-A, Saldūnaite K. Severe early childhood caries and behavioral risk factors among 3-year-old children in Lithuania. Medicina. 2010;46(2):135.

45. Zhou Y, Lin H, Lo E, Wong M. Risk indicators for early childhood caries in 2year-old children in southern China. Aust Dent J. 2011;56(1):33-9.

46. Rajshekar SA, Laxminarayan N. Comparison of primary dentition caries experience in pre-term low birth-weight and full-term normal birth-weight children aged one to six years. J Indian Soc Pedod Prev Dent. 2011;29(2):128.

47. Ozer S, Sen Tunc E, Bayrak S, Egilmez T. Evaluation of certain risk factors for early childhood caries in Samsun, Turkey. Eur J Paediatr Dent. 2011;12(2): 103.

48. Li Y, Zhang Y, Yang R, Zhang Q, Zou J, Kang D. Associations of social and behavioural factors with early childhood caries in Xiamen city in China. Int J Paediatr Dent. 2011:21(2):103-11.

49. Kumarihamy SL, Subasinghe LD, Jayasekara P, Kularatna SM, Palipana PD. The prevalence of early childhood caries in 1-2 yrs olds in a semi-urban area of Sri Lanka. BMC Res Notes. 2011;4(1):336.
50. Prakash P, Subramaniam P, Durgesh B, Konde S. Prevalence of early childhood caries and associated risk factors in preschool children of urban Bangalore, India: a cross-sectional study. Eur J Dent. 2012;6(2):141.

51. Singh S, Vijayakumar N, Priyadarshini H, Shobha M. Prevalence of early childhood caries among 3-5 year old pre-schoolers in schools of Marathahalli, Bangalore. Dental Res J. 2012;9(6):710.

52. Perera PJ, Abeyweera NT, Fernando MP, Warnakulasuriya TD, Ranathunga N. Prevalence of dental caries among a cohort of preschool children living in Gampaha district, Sri Lanka: a descriptive cross sectional study. BMC Oral Health. 2012;12(1):49.

53. Phipps KR, Ricks TL, Manz MC, Blahut P. Prevalence and severity of dental caries among American Indian and Alaska native preschool children. J Public Health Dent. 2012;72(3):208-15.

54. Parisotto TM, Steiner-Oliveira C, De Souza-e-Silva CM, Peres RC, Rodrigues LK, Nobre-Dos-Santos M. Assessment of cavitated and active non-cavitated caries lesions in 3-to 4-year-old preschool children: a field study. Int J Paediatr Dent. 2012:22(2):92-9.

55. Zhang S, Liu J, Lo EC, Chu C-H. Dental caries status of Bulang preschool children in Southwest China. BMC Oral Health. 2014;14(1):16.

56. Tanaka K, Miyake Y. Low birth weight, preterm birth or small-for-gestationalage are not associated with dental caries in young Japanese children. BMC Oral Health. 2014;14(1):38.

57. Colombo S, Gallus S, Beretta M, Lugo A, Scaglioni S, Colombo P, Paglia M, Gatto R, Marzo G, Caruso S. Prevalence and determinants of early childhood caries in Italy. Eur J Paediatr Dent. 2019;20(4):267.

58. Agouropoulos A, Birpou E, Twetman S, Kavvadia K. Validation of three caries risk assessment tools for preschool children from areas with high caries prevalence. Pediatr Dent. 2019;41(5):391-9.

59. Musinguzi N, Kemoli AM, Okullo I. Prevalence and treatment needs for early childhood caries among 3-5-year-old children from a rural community in Uganda. Front Public Health. 2019;7:259.

60. Montes GR, Bonotto DV, Ferreira FM, Menezes JVNB, Fraiz FC. Caregiver's oral health literacy is associated with prevalence of untreated dental caries in preschool children. Cien Saude Colet. 2019;24(7):2737-44.

61. Boustedt K, Dahlgren J, Twetman S, Roswall J. Tooth brushing habits and prevalence of early childhood caries: a prospective cohort study. Eur Arch Paediatric Dent. 2019:1-5.

62. Tonpe M, Patil RU, Kadam A, Bayad P, Shetty V, Vinay V. Comparative evaluation of two caries detection systems for detecting the prevalence of early childhood caries: a cross-sectional study. Dental Res J. 2019;16(4):221.

63. Wang Z, Rong W, Zhang Y, Zeng X, Li Z, Liu Z. Prevalence and contributing factors of dental caries of 6-year-old children in four regions of China. PeerJ. 2019;7:e6997.

64. Nomura Y, Maung K, Khine K, Min E, Sint KM, Lin MP, Myint W, Khaing M, Aung T, Sogabe K. Prevalence of dental caries in 5-and 6-year-old Myanmar children. Int J Dent. 2019:2019:5948379.

65. Wu X, Wang J, Cai T, Li Y, Zhou Z, Yang Z. Prevalence and influencing factors of deciduous caries in preschool children in Chongqing city. Hua Xi Kou Qiang Yi Xue Za Zhi. 2019;37(1):81-6.

66. Abbass MM, Mahmoud SA, El Moshy S, Rady D, AbuBakr N, Radwan IA, Ahmed A, Abdou A, Al Jawaldeh A. The prevalence of dental caries among Egyptian children and adolescences and its association with age, socioeconomic status, dietary habits and other risk factors. A cross-sectional study. F1000Research. 2019;8:8.

67. Goenka P, Dutta S, Marwah N, Sarawgi A, Nirwan M, Mishra P. Prevalence of dental caries in children of age 5 to 13 years in district of Vaishali, Bihar, India. Int J Clin Pediatr Dent. 2018;11(5):359.

68. Chugh VK, Sahu KK, Chugh A. Prevalence and risk factors for dental caries among preschool children: a cross-sectional study in eastern India. Int J Clin Pediatr Dent. 2018;11(3):238.

69. Vandana K, Raju SH, Badepalli RR, Narendrababu J, Reddy C, Sudhir K. Prevalence and risk-factors of early childhood caries among 2-6-year-old Anganwadi children in Nellore district, Andhra Pradesh, India: a crosssectional survey. Indian J Dent Res. 2018;29(4):428.

70. Igic M, Obradovic R, Filipovic G. Prevalence and progression of early childhood caries in Nis, Serbia. Eur J Paediatr Dent. 2018;19(2):161-4.

71. Kato H, Tanaka K, Shimizu K, Nagata C, Furukawa S, Arakawa M, Miyake Y. Parental occupations, educational levels, and income and prevalence of dental caries in 3-year-old Japanese children. Environ Health Prev Med. 2017:22(1):80.

72. Li Y, Wulaerhan J, Liu Y, Abudureyimu A, Zhao J. Prevalence of severe early childhood caries and associated socioeconomic and behavioral factors in Xinjiang, China: a cross-sectional study. BMC Oral Health. 2017;17(1):144 
73. Mangla RG, Kapur R, Dhindsa A, Madan M. Prevalence and associated risk factors of severe early childhood caries in 12-to 36-month-old children of Sirmaur district, Himachal Pradesh, India. Int J Clin Pediatr Dent. 2017;10(2): 183.

74. Owen M, Ghanim A, Elsby D, Manton D. Hypomineralized second primary molars: prevalence, defect characteristics and relationship with dental caries in Melbourne preschool children. Aust Dent J. 2018;63(1):72-80.

75. Pal A, Gupta S, Rao A, Kathal S, Roy S, Pandey S. Family-related factors associated with caries prevalence in the primary dentition of 5-6-year-old children in urban and rural areas of Jabalpur City. Contemp Clin Dent. 2017; 8(2):305.

76. Wagne $Y$, Heinrich-Weltzien R. Caries prevalence and risk assessment in Thuringian infants, Germany. Oral Health Prev Dent. 2017;15(5):489-94.

77. Shah N, Mathur VP, Kant S, Gupta A, Kathuria V, Haldar P, Pandey RM. Prevalence of dental caries and periodontal disease in a rural area of Faridabad District, Haryana, India. Indian J Dent Res. 2017;28(3):242.

78. Yuan S, Shi L, LV J. Survey on dental caries prevalence among 3-year-old children in Jing'an district of Shanghai. Shanghai Kou Qiang Yi Xue. 2017; 26(1):118-20.

79. Jiang $Y-Y$, Jiang $Y-Y$. Prevalence of early childhood caries among 2-to 5year-old preschoolers in kindergartens of Weifang City, China: a crosssectional study. Oral Health Prev Dent. 2017;15(1):89-97.

80. Massignan C, Ximenes M, da Silva Pereira C, Dias L, Bolan M, Cardoso M. Prevalence of enamel defects and association with dental caries in preschool children. Eur Arch Paediatr Dent. 2016;17(6):461-6.

81. Koya S, Ravichandra K, Arunkumar VA, Sahana S, Pushpalatha H. Prevalence of early childhood caries in children of west Godavari District, Andhra Pradesh, South India: an epidemiological study. Int J Clin Pediatr Dent. 2016; 9(3):251.

82. Mothupi KA, Nqcobo CB, Yengopal V. Prevalence of early childhood caries among preschool children in Johannesburg, South Africa. J Dent Child. 2016:83(2):83-7

83. Alkhtib A, Ghanim A, Temple-Smith M, Messer LB, Pirotta M, Morgan M. Prevalence of early childhood caries and enamel defects in four and fiveyear old Qatari preschool children. BMC Oral Health. 2016;16(1):73.

84. Henry JA, Muthu MS, Saikia A, Asaithambi B, Swaminathan K. Prevalence and pattern of early childhood caries in a rural south Indian population evaluated by ICDAS with suggestions for enhancement of ICDAS software tool. Int J Paediatr Dent. 2017;27(3):191-200.

85. Šačić L, Marković N, Muratbegović AA, Zukanović A, Kobašlija S. The prevalence and severity of early childhood caries in preschool children in the Federation of Bosnia and Herzegovina. Acta Med Acad. 2016;45(1):19.

86. Al-Meedani LA, Al-Dlaigan YH. Prevalence of dental caries and associated social risk factors among preschool children in Riyadh, Saudi Arabia. Pak J Med Sci. 2016:32(2):452.

87. Elidrissi SM, Naidoo S. Prevalence of dental caries and toothbrushing habits among preschool children in Khartoum state, Sudan. Int Dent J. 2016;66(4): 215-20

88. Gopal S, Chandrappa V, Kadidal U, Rayala C, Vegesna M. Prevalence and predictors of early childhood caries in 3-to 6-year-old south Indian children--a cross-sectional descriptive study. Oral Health Prev Dent. 2016; 14(3):267-73.

89. Aghighi S, Omrani L. Evaluation of DMFT index and level of teeth and mouth hygiene education among students in air force military bases in the year 2008-2009. EBNESINA. 2010;12(4):15-9.

90. Mortazavi M, Ebrahimi Z. The prevalence of dental decay in 6 to 9 year old children in shiraz. J Dent. 1997;9(3,4):69-81 [In Persian].

91. Asdagh S, Nuroloyuni S, Amani F, Sadeghi MT. Dental caries prevalence among 6-12 years old school children in Ardabil city, 2012; 2015.

92. Memar N, Ghazizade A, Mahmoudi SH. DMFT index (decayed-missing-filled Teath) and its effective factors in 12-year-old students in Sanandaj. Kurdistan Med J. 1999;5(17):26-31 [In Persian].

93. Javadine Jad SH, Karami M, Azizi HR. Caries prevalence in 12-year -o ld children of I sfahan City expressed by the significant caries index. MUI. 2006; 2(2):13-7 [In Persian].

94. Sadeghi M. Prevalence and bilateral occurrence of first permanent molar caries in 12-year-old students. J Dent Res Dent Clin Dental Prospects. 2007; 1(2):86.

95. Yousofi M, Behrouzpour K, Kazemi S, Afroughi S. Dental caries and related factors among 7-12 year-old school children in Yasuj, Iran, in 2014. Armaghane Danesh. 2015;20(9):836-47.
96. Eskandarizadeh A, Sajadi FS, Torabi M, Sharifi M, Amini Z, Sahebghalam B, Mahdavi SS, Asadpoor S, Ehsan N, Saeedi V. Caries free prevalence among 6 12 \& 15-year old school children in Kerman during 2000-2005. J Health Dev. 2015:4(1):42-51.

97. Mossaheb P, Abadi A, Amini M. The relationship between food intake and dental caries in a group of Iranian children in 2009. Res Dent Sci. 2011;7(4): $50-42$.

98. Qin D, Jiang HF, Shen L, Zhang C, Chai ZW, Wang JH. Prevalence of dental caries and associated factors among 10-12-year-old students in Chongqing. Hua Xi Kou Qiang Yi Xue Za Zhi. 2019;37(6):608-14.

99. Alshehhi A, Al Halabi M, Hussein I, Salami A, Hassan A, Kowash M. Enamel defects and caries prevalence in preterm children aged 5-10 years in Dubai. Libyan J Med. 2020;15(1):1705633.

100. Cheng Y-H, Liao Y, Chen D-Y, Wang Y, Wu Y. Prevalence of dental caries and its association with body mass index among school-age children in Shenzhen, China. BMC Oral Health. 2019;19(1):270.

101. Villanueva-Gutiérrez T, Irigoyen-Camacho ME, Castaño-Seiquier A, ZepedaZepeda MA, Sanchez-Pérez L, Frechero NM. Prevalence and severity of molar-incisor hypomineralization, maternal education, and dental caries: a cross-sectional study of Mexican schoolchildren with low socioeconomic status. J Int Soc Prev Community Dent. 2019;9(5):513.

102. Lešić S, Dukić W, Šapro KZ, Tomičić V, Kadić S. Caries prevalence among schoolchildren in urban and rural Croatia. Cent Eur J Public Health. 2019; 27(3):256.

103. Mohd Nor NA, Chadwick B, Farnell D, Chestnutt I. The prevalence of enamel and dentine caries lesions and their determinant factors among children living in fluoridated and non-fluoridated areas. Community Dent Health. 2019;36(3):229-36.

104. Vanvitelli CL. Prevalence of caries and dental malocclusions in the apulian paediatric population: an epidemiological study. Eur J Paediatr Dent. 2019. 20:100.

105. Obregón-Rodríguez N, Fernández-Riveiro P, Piñeiro-Lamas M, SmythChamosa E, Montes-Martínez A, Suárez-Cunqueiro M. Prevalence and cariesrelated risk factors in schoolchildren of 12-and 15-year-old: a cross-sectional study. BMC Oral Health. 2019;19(1):120.

106. Mimoza C, Vito MA. Evaluation of caries prevalence and decayed-, missing-, and filled-teeth values in permanent dentition in children 7 to 10 years olda longitudinal study. J Contemp Dent Pract. 2019;20(1):8-12.

107. Aldossary MS, Alamri AA, Alshiha SA, Hattan MA, Alfraih YK, Alwayli HM. Prevalence of dental caries and fissure sealants in the first permanent molars among male children in Riyadh, Kingdom of Saudi Arabia. Int J Clin Pediatr Dent. 2018;11(5):365.

108. Ballouk MA-H, Dashash M. Caries prevalence and dental health of 8-12 year-old children in Damascus city in Syria during the Syrian crisis; a crosssectional epidemiological oral health survey. BMC Oral Health. 2019;19(1):16.

109. Alhabdan YA, Albeshr AG, Yenugadhati N, Jradi H. Prevalence of dental caries and associated factors among primary school children: a populationbased cross-sectional study in Riyadh, Saudi Arabia. Environ Health Prev Med. 2018;23(1):60.

110. Konde S, Darshini CS, Agarwal M, Peethambar P. Unrevealed caries in unerupted teeth: a prevalence study. Contemp Clin Dent. 2018;9(Suppl 2): S305

111. Solis-Riggioni A, Gallardo-Barquero C, Chavarria-Bolaños D. Prevalence and severity of dental caries in foster-care children and adolescents. J Clin Pediatr Dent. 2018;42(4):269-72.

112. Musa TH, Li W, Li X, Wang WX, Soro WL, Gao R, Song Y, He Y, Hong L, Musa $\mathrm{HH}$. Prevalence of dental caries profile in children and adolescents in rural Jiangsu Province. Arch Dis Child. 2018;103(12):1184-5.

113. Dutra ER, Chisini LA, Cademartori MG, Oliveira LJC d, Demarco FF, Correa MB. Accuracy of partial protocol to assess prevalence and factors associated with dental caries in schoolchildren between 8-12 years of age. Cad Saude Publica. 2018;34:e00077217.

114. Al-Akwa AA, Al-Maweri SA. Dental caries prevalence and its association with fluoride level in drinking water in Sana'a, Yemen. Eur J Dent. 2018:12(1):15.

115. Cruz MGB d, Narvai PC. Caries and fluoridated water in two Brazilian municipalities with low prevalence of the disease. Rev Saude Publica. 2018; 52:28

116. Andegiorgish AK, Weldemariam BW, Kifle MM, Mebrahtu FG, Zewde HK, Tewelde MG, Hussen MA, Tsegay WK. Prevalence of dental caries and associated factors among 12 years old students in Eritrea. BMC Oral Health. 2017;17(1):169. 
117. Alwayli HM, Alshiha SA, Alfraih YK, Hattan MA, Alamri AA, Aldossary MS. A survey of fissure sealants and dental caries prevalence in the first permanent molars among primary school girls in Riyadh, Saudi Arabia. Eur J Dent. 2017:11(4):455

118. Dobbiani A, Berton F, Perinetti G, Costantinides F, Di RL. Prevalence of dental caries among schoolchildren from north-eastern Italian population. Minerva Stomatol. 2018;67(2):49-54

119. Maran S, Shashikiran N, Ahirwar P, Maran P, Kannojiya PR, Niranjan B. Prevalence of dental caries and traumatic dental injuries among 6-to 12year-old children in Bhopal City, India. Int J Clini Pediatr Dent. 2017;10(2): 172.

120. Kim H-N, Kim J-H, Kim S-Y, Kim J-B. Associations of community water fluoridation with caries prevalence and oral health inequality in children. Int J Environ Res Public Health. 2017;14(6):631.

121. Sköld U. Approximal caries increment in relation to baseline approximal caries prevalence among adolescents in Sweden with and without a school-based fluoride varnish programme. Community Dent Health. 2016; 33(4):281-5

122. Plaka K, Ravindra K, Mor S, Gauba K. Risk factors and prevalence of dental fluorosis and dental caries in school children of North India. Environ Monit Assess. 2017;189(1):40.

123. Hiremath A, Murugaboopathy V, Ankola AV, Hebbal M, Mohandoss S, Pastay P. Prevalence of dental caries among primary school children of India-a cross-sectional study. J Clin Diagn Res. 2016;10(10):ZC47.

124. Kottayi S, Bhat S, Hegde K, Peedikayil F, Chandru T, Anil S. A cross-sectional study of the prevalence of dental caries among 12-to 15-year-old overweight schoolchildren. J Contemp Dent Pract. 2016;17(9):750-4.

125. Ponnudurai Arangannal SKM, Jayaprakash J. Prevalence of dental caries among school children in Chennai, based on ICDAS II. J Clin Diagn Res. 2016;10(4):ZC09.

126. Djossou D, Nancy J, Houinato D, Lanchoessi D. Prevalence of dental caries in school in the city of Ouidah in 2013. Odonto-stomatol Trop. 2015;38(150): $15-22$.

127. Weusmann J, Mahmoodi B, Azaripour A, Kordsmeyer K, Walter C, Willershausen B. Epidemiological investigation of caries prevalence in first grade school children in Rhineland-Palatinate, Germany. Head Face Med. 2015;11(1):33.

128. Goel R, Vedi A, Veeresha K-L, Sogi G-M, Gambhir R-S. Oral hygiene practices and dental caries prevalence among $12 \& 15$ years school children in Ambala, Haryana-a cross-sectional study. J Clin Exp Dent. 2015;7(3):e374.

129. Farooqi FA, Khabeer A, Moheet IA, Khan SQ, Farooq I, ArRejaie AS. Prevalence of dental caries in primary and permanent teeth and its relation with tooth brushing habits among schoolchildren in eastern Saudi Arabia. Saudi Med J. 2015;36(6):737-42.

130. Arora G, Bhateja S. Prevalence of dental caries, periodontitis, and oral hygiene status among 12-year-old schoolchildren having normal occlusion and malocclusion in Mathura city: a comparative epidemiological study. Indian J Dent Res. 2015;26(1):48.

131. Sukhabogi JR, Parthasarathi P, Anjum S, Shekar B, Padma C, Rani A. Denta fluorosis and dental caries prevalence among 12 and 15 year old school children in Nalgonda district, Andhra Pradesh, India. Ann Med Health Sci Res. 2014;4(3):245-52.

132. Al-Darwish M, El Ansari W, Bener A. Prevalence of dental caries among 1214 year old children in Qatar. Saudi Dent J. 2014;26(3):115-25.

133. Aidara A, Bourgeois D. Prevalence of dental caries: national pilot study comparing the severity of decay (CAO) vs ICDAS index in Senegal. Odontostomatol Trop. 2014;37(145):53-63.

134. Ingle NA, Dubey HV, Kaur N, Gupta R. Prevalence of dental caries among school children of Bharatpur city, India. J Int Soc Prev Community Dent. 2014:4(1):52

135. Sofola O, Folayan M, Oginni A. Changes in the prevalence of dental caries in primary school children in Lagos state, Nigeria. Niger J Clin Pract. 2014; 17(2):127-33.

136. Das D, Misra J, Mitra M, Bhattacharya B, Bagchi A. Prevalence of dental caries and treatment needs in children in coastal areas of West Bengal. Contemp Clin Dent. 2013:4(4):482

137. Mahfouz M, Esaid AA. Dental caries prevalence among 12-15 year old Palestinian children. Int Sch Res Notices. 2014;2014:785404.

138. Riziwaguli A, Asiya Y, Liu Y, Yang R, Zou J. Caries prevalence of the first permanent molar among 7-9 years old Uygur children in Urumqi, Xinjiang autonomous region. Shanghai Kou Qiang Yi Xue. 2013;22(5):559-61.
139. Joshi N, Sujan S, Joshi K, Parekh H, Dave B. Prevalence, severity and related factors of dental caries in school going children of Vadodara city-an epidemiological study. J Int Oral Health. 2013;5(4):35.

140. Pieper K, Lange J, Jablonski-Momeni A, Schulte A. Caries prevalence in 12 year-old children from Germany: results of the 2009 national survey. Community Dent Health. 2013;30(3):138-42.

141. Yengopal V, Nqcobo C, Thekiso M, Rudolph M, Joosab Z. Dental caries prevalence in children attending special needs schools in Johannesburg, Gauteng Province, South Africa. South Afr Dent J. 2012;67(7):308-13.

142. Murthy A, Pramila M, Ranganath S. Prevalence of clinical consequences of untreated dental caries and its relation to dental fear among 12-15-year-old schoolchildren in Bangalore city, India. Eur Arch Paediatr Dent. 2014;15(1): 45-9.

143. Koposova N, Eriksen HM, Widstrom E, Handegard B, Pastbin M, Koposov R. Caries prevalence and determinants among 12-year-olds in north-West Russia and northern Norway. Stomatologija. 2013;15(1):3-11.

144. Dixit A, Hao F, Mukherjee S, Lakshman T, Kompella R. Towards an elastic distributed SDN controller. ACM SIGCOMM Comput Commun Rev. 2013, 43(4):7-12.

145. Suprabha BS, Rao A, Shenoy R, Khanal S. Utility of knowledge, attitude, and practice survey, and prevalence of dental caries among 11-to 13-year-old children in an urban community in India. Glob Health Action. 2013;6(1): 20750.

146. Panagidis D, Schulte A. Caries prevalence in 12-year-old Cypriot children. Community Dent Health. 2012;29(4):297.

147. Shailee F, Sogi G, Sharma K, Nidhi P. Dental caries prevalence and treatment needs among 12-and 15-year old schoolchildren in Shimla city, Himachal Pradesh, India. Indian J Dent Res. 2012;23(5):579.

148. Lagana G, Fabi F, Abazi Y, Kerçi A, Jokici M, Nastasi EB, Vinjolli F, Cozza P. Caries prevalence in a 7-to 15-year-old Albanian schoolchildren population. Ann Stomatol. 2012;3(2):38

149. Subedi B, Shakya P, Kc U, Jnawali M, Paudyal B, Acharya A, Koirala S, Singh A Prevalence of dental caries in 5-6 years and 12-13 years age group of school children of Kathmandu valley. J Nepal Med Assoc. 2011;51(184):176-81.

150. Oulis CJ, Berdouses ED, Mamai-Homata E, Polychronopoulou A. Prevalence of sealants in relation to dental caries on the permanent molars of 12 and 15-year-old Greek adolescents. A national pathfinder survey. BMC Public Health. 2011:11(1):100.

151. Jamelli SR, Rodrigues CS, de Lira PIC. Nutritional status and prevalence of dental caries among 12-year-old children at public schools: a case-control study. Oral Health Prev Dent. 2010;8(1):77-84.

152. Kanagaratnam S, Schluter P, Durward C, Mahood R, Mackay T. Enamel defects and dental caries in 9-year-old children living in fluoridated and nonfluoridated areas of Auckland, New Zealand. Community Dent Oral Epidemiol. 2009:37(3):250-9.

153. Bissar A-R, Schulte AG, Muhjazi G, Koch MJ. Caries prevalence in 11-to 14year old migrant children in Germany. Int J Public Health. 2007:52(2):103-8.

154. Ferro R, Besostri A, Meneghetti B, Stellini E. Prevalence and severity of dental caries in 5-and 12-year old children in the Veneto region (Italy). Community Dent Health. 2007;24(2):88.

155. Moreira PVL, Rosenblatt A, Severo AMR. Prevalence of dental caries in obese and normal-weight Brazilian adolescents attending state and private schools. Community Dent Health. 2006;23(4):251.

156. Schulte AG, Momeni A, Pieper K. Caries prevalence in 12-year-old children from Germany. Results of the 2004 national survey. Community Dent Health. 2006;23(4):197.

157. Paredes VG, Paredes CC, Mir BP. Prevalence of dental caries: comparison between immigrant and autochthonous children. An Pediatr (Barc). 2006; 65(4):337-41.

158. Mestriner SF, Pardini LC, Mestriner WJ. Impact of the bitewing radiography exam inclusion on the prevalence of dental caries in 12-year-old students in the city of Franca, São Paulo, Brazil. J Appl Oral Sci. 2006;14(3):167-71.

159. Traebert J, Suárez CS, Onofri DA, Marcenes W. Prevalence and severity of dental caries and treatment needs in small Brazilian counties. Cad Saude Publica. 2002;18(3):817-21.

160. Nag R, Bihani VK, Panwar VR, Acharya J, Bihani T, Pandey R. Prevalence of dental caries and treatment needs in the school going children in Bikaner, Rajasthan-an observational study. J Indian Dent Assoc. 2012;6(1):12.

161. Kumar S, Tadakamadla J, Kroon J, Johnson NW. Impact of parent-related factors on dental caries in the permanent dentition of 6-12-year-old children: a systematic review. J Dent. 2016;46:1-11. 
162. Hermes Soares G, Fernanda Pereira N, Gabriela Haye Biazevic M, Minatel Braga M, Michel-Crosato E. Dental caries in south American indigenous peoples: a systematic review. Community Dent Oral Epidemiol. 2019;47(2): $142-52$.

163. Marghalani AA, Guinto E, Phan M, Dhar V, Tinanoff N. Effectiveness of xylitol in reducing dental caries in children. Pediatr Dent. 2017;39(2):103-10.

164. Hegde MN, Attavar SH, Shetty N, Hegde ND, Hegde NN. Saliva as a biomarker for dental caries: a systematic review. J Conserv Dent. 2019;22(1): 2-6.

165. Masaheb P, Kargarnovin Z, Malekafzali B, Abadi AR, Amini M. The relationship between food intake and dental caries in a group of Iranian children in 2009. J Res Dent Sci. 2011;7(4):50-42.

166. Prakash P, Subramaniam P, Durgesh BH, Konde S. Prevalence of early childhood cariesand associated risk factors in preschool children of urban Bangalore, India: a cross-sectional study. Eur J Dent. 2012:6(2):141-52.

167. Namal N, Yuceokur AA, Can G. Significant caries index values and related factors in 5-6-year-old children in Istanbul, Turkey. East Mediterr Health J. 2009;15(1):178-84.

168. Wagner $Y$, Heinrich-Weltzien R. Caries prevalence and risk assessment in Thuringian infants, Germany. Oral Health Prev Dent. 2017;25:1-6.

\section{Publisher's Note}

Springer Nature remains neutral with regard to jurisdictional claims in published maps and institutional affiliations.

Ready to submit your research? Choose BMC and benefit from:

- fast, convenient online submission

- thorough peer review by experienced researchers in your field

- rapid publication on acceptance

- support for research data, including large and complex data types

- gold Open Access which fosters wider collaboration and increased citations

- maximum visibility for your research: over $100 \mathrm{M}$ website views per year

At BMC, research is always in progress.

Learn more biomedcentral.com/submissions 